\title{
Shielding factors for gamma radiation from activity deposited on structures and ground surfaces
}

\author{
Jensen, Per Hedemann
}

Publication date:

1982

Document Version

Publisher's PDF, also known as Version of record

Link back to DTU Orbit

Citation $(A P A)$ :

Jensen, P. H. (1982). Shielding factors for gamma radiation from activity deposited on structures and ground surfaces. Risø National Laboratory. Risø-M No. 2270

\section{General rights}

Copyright and moral rights for the publications made accessible in the public portal are retained by the authors and/or other copyright owners and it is a condition of accessing publications that users recognise and abide by the legal requirements associated with these rights.

- Users may download and print one copy of any publication from the public portal for the purpose of private study or research.

- You may not further distribute the material or use it for any profit-making activity or commercial gain

- You may freely distribute the URL identifying the publication in the public portal

If you believe that this document breaches copyright please contact us providing details, and we will remove access to the work immediately and investigate your claim. 
Rise-M-2270

SHIELDING FACTORS FOR GAMMA RADIATION FROM ACTIVITY DEPOSITED ON STRUCTURES AND GROUND SURFACES.

Per Hedemann Jensen

Abstract. This report describes a computer model that calculates shielding factors for indoor residence in multistorey and single-family houses for gamma radiation from activity deposited on roofs, outer walls, and ground surfaces. The dimensions of the buildings including window areas and the nearby surroundings has to be specified in the calculations.

3hielding factors can he calculated for different photon energies and for a uniform surface activity distribution as well as for separate activity on roof, outer wall, and ground surface achieved from decontamination or different deposition velocities.

For a given area with a known distribution of different houses a weighted shielding factor can be calculated as well as a timeaveraged one based on a given residence time distribution for work/school, home, outdoors, and transportation.

Calculated shielding factors are shown for typical Danish houses. To give an impression of the sensitivity of the shielding factors on the parameters used in the model, vari(Continued on nest page)

November 1982

Risø National Laboratory, DK-4000 Roskilde, Denmark 
ations were made in some of the most important parameters: wall thickness, road and qround width, percentage of outer wall covered by windows, photon energy, and decontamination percentage for outer walls, ground and roofs. The uncertainty of the calculations is discussed.

INIS describtors: BUILDINGS; COMPUTER CALCULATIONS; DECONTAMINATION; DOSE RATES; EXTERNAL IRRADIATION; FALLOUT DEPOSITS; GAMMA RADIATION; RADIATION DOSES; SHIELDING; SURFACE CONTAMINATION; SURFACES

UDC $539.166: 614.876$

ISBN 87-550-0933-6

ISSN 0418-6435

Riso repro 1983 
1. INTRODUCTION $\ldots \ldots \ldots \ldots \ldots \ldots \ldots \ldots \ldots \ldots \ldots \ldots \ldots \ldots \ldots \ldots \ldots$

2. DOSE RATE FROM SURFACE SOURCES $\ldots \ldots \ldots \ldots \ldots \ldots \ldots \ldots \ldots$. 6

2.1. Outdoor dose rate .................... 6

2.1.1. Dose rate from an infinite surface ......6 6

2.1.2. Dose rate from a finite road surface ..... 8

2.1.3. Dose rate from outer walls ............ 9

2.2. Indoor dose rate $\ldots \ldots \ldots \ldots \ldots \ldots \ldots \ldots \ldots \ldots \ldots$

2.2.1. Dose rate from a finite road surface .... 10

2.2.2. Dose rate from outer walls ........... 14

2.2.3. Dose rate from a roof ............... 16

3. SHIELDING FACTORS FOR BUILDINGS .............. 18

3.1. Definition of the shielding factor .......... 18

3.2. Outdoor shielding factor ................. 18

3.3. Shielding factor for a single residence ....... 19

3.4. Weighted shielding factor ................. 19

3.5. Time-averaged shielding factor ............. 20

3.6. Calculated shielding factors for Danish houses .. 21

3.6.1. Attenuation and build-up data for building

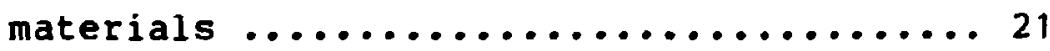

3.6.2. Shielding factors for typical buildings .. 23

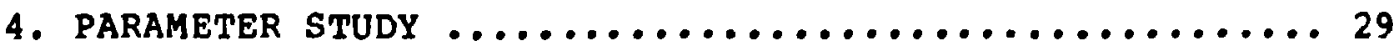

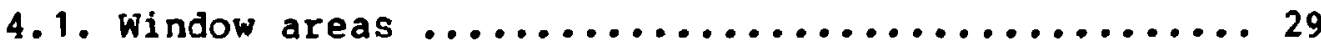

4.2. Outer-wall thickness $\ldots \ldots \ldots \ldots \ldots \ldots \ldots \ldots \ldots \ldots \ldots$

4.3. Inner-wall thickness ................... 33

4.4. Road and ground width ................... 34

4.5. Decontamination of surfaces .............. 35

4.6. Photon energy ....................... 39

5. DISCUSSION OF UNCERTAINTIES ................ 40

6. CONCLUSION .............................. 42

7. REFERENCES ................................... 44 



\section{INTRODUCTION}

In studies of the potential radiological consequences from hypothetical nuclear reactor accidents where radioactivity is released to the atmosphere, attention has in the last few years been qiven to the long-term radiation doses originating from activity deposited on structures and ground surfaces. In particular, the discussions have focused on sites where nuclear power plants have been placed near large population centers giving a potential long-term exposure risk from radionuclides such as $137 \mathrm{Cs}$.

The gamma dose rate at a given indoor residence from deposited activity will depend on the activity concentration from deposition on outer walls, roofs, and the surrounding ground surfaces, as well as the building construction regarding building size, position of the apartment, wingow areas, and thickness of walls, floors and roofs.

In calculations of the dose rate from deposited activity most computer models use the dose rate one meter above an infinite smooth, plane source as a reference. The actual dose rate at a given location is then found by multiplying this reference dose rate with a modifying factor - the so-called shielding factor.

The shielding factor is usually defined as the ratio of the indoor dose rate from deposited activity on an infinite ground surface to the reference dose rate noted atove.

To make more realistic dose calculations for urban areas a computer model has been developed for calculating shielding factors that also include radiation from deposited activity on outer walls and roofs, and which consider the finite size of the surrounding ground surface. 
The main feature of the model is that the activity concentration could differ at the various surfaces. Therefore, shielding f. ctors can be calculated for houses where the deposition velocity to the ground surface differs from that of the house surfaces, as well as for situations where decontamination has changed the surface activity distribution.

In risk studies, where the long-term stochastic consequences from lijing in contaminated urban areas are estimated, it is important to know the effect of a given decontamination measure. For this purpose the present model can be used to give an improved I -cture for urban areas than previousiy achieved.

2. DOSE RATE FROH SURFACE SOURCES

2.1. Outdoor dose rate

2.1.1._Dose rate_from an infinite surface

The photon flux density from an infinite plane surfase source having the source concentration $Q$ can be described in the following way with reference to figure $1:$

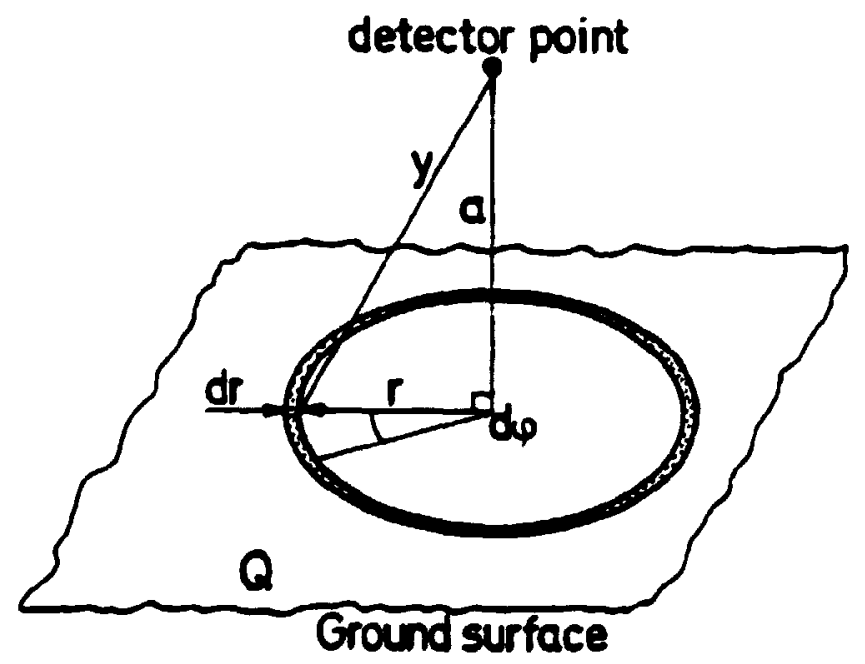

Fig. 1. Geometry for calculating the dose rate from an infinite plane surface source. 
The activity in the infinitesinal ring element with radius from $r$ to $r+d r$ is:

$$
d q=2 \pi \cdot Q \cdot r \cdot d r
$$

From the source point to the detector point the photons will experience build-up as well as attenuation in air.

With regard to this, the photon flux density at the detector point from the ring element is given by:

$$
\text { defef }=2 \pi \cdot \frac{Q \cdot r \cdot d r}{4 \pi \cdot y^{2}} \cdot B\left(\mu_{a i r} \cdot y\right) \cdot e^{-\mu_{a i r} y}
$$

where $y=\sqrt{r^{2}+a^{2}}$, B is the dose build-up factor in air, and Mair is the linear attenuation coefficient for air.

The total photon flux density is then:

$$
\Phi_{\text {ref }}=\frac{Q}{2} \cdot \int_{0}^{\infty} \frac{r}{r^{2}+a^{2}} \cdot B\left(\mu_{a i r} Y\right) \cdot e^{-\mu_{a i r} Y} d r
$$

The dose rate is given by:

$$
\begin{aligned}
& \dot{D}_{\text {ref }}=\left(\frac{\mu_{e n}}{\rho}\right)_{\text {air }} \cdot \omega_{\text {ref }} \cdot E \\
& =\frac{Q}{2} \cdot\left(\frac{\mu_{e n}}{\rho}\right)_{\text {air }} \cdot \int_{0}^{-} \frac{r}{r^{2}+a^{2}} \cdot B\left(\mu_{a i r} y\right) \cdot e^{-\mu_{a i r} y} d r
\end{aligned}
$$

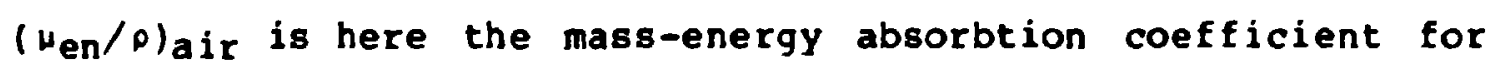
air and $E$ is the photon energy.

This dose rate is used as reference dose rate for the calculation of shielding factors in section 3 . 


\section{$\underline{2} \cdot 1 \cdot \underline{2} \cdot$-Dose rate_from a finite_road surface}

The photon flux density from a plane rectangular source with the concentration $Q$, length $2 L$ and width $w$ is calculated as follows with reference to Figure $2:$

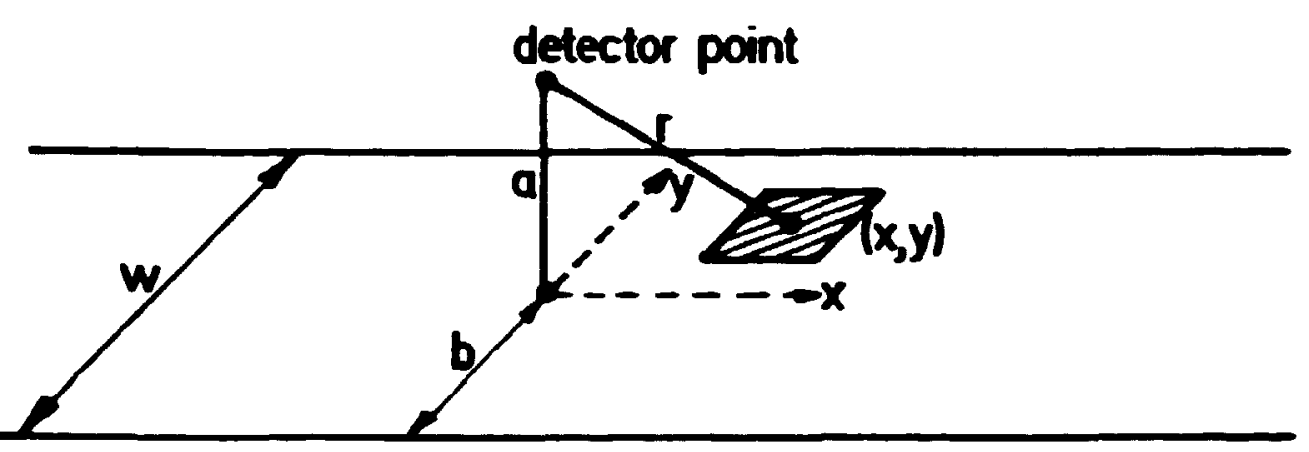

Fig. 2. Geometry for calculating the dose rate from a road of length $2 \mathrm{~L}$ and width w.

The activity in an infinitesimal ground element around $(x, y)$ is:

$$
d q=Q \cdot d x \cdot d y
$$

Taking build-up and attenuation in air into consideration gives the follo:ing expression for the photon flux density at the detector point from the ground element:

$$
\begin{aligned}
& d *_{\text {ground }}=\frac{Q \cdot d x \cdot d y}{4 \pi r^{2}} \cdot B\left(\mu_{\text {air }} r\right) \cdot e^{-\mu_{a i r} \cdot r} \\
& r^{2}=x^{2}+y^{2}+a^{2}
\end{aligned}
$$

This gives the total flux density as:

$$
\text { ground }=\int_{-L}^{L} \int_{-b}^{W-b} \frac{Q}{4 \pi r^{2}} B\left(\mu_{a i r} r\right) \cdot e^{-\mu_{a i r} r} d y \cdot d x
$$

The outdoor dose rate at the distance a above the road and distance b from the road side is then: 


$$
\dot{D}_{\text {out }, g}=\frac{Q}{4 \pi} \cdot\left(\frac{\mu_{e n}}{p}\right)_{a i r} \cdot \int_{-L}^{L} \int_{-b}^{w-b} \frac{B\left(\mu_{a i r} \cdot x\right)}{r^{2}} \cdot e^{-\mu_{a i r} \cdot r} d y \cdot d x
$$

where $E$ and $\mathrm{Hen}_{\mathrm{p}}$ are the sane as defined in Sec. 2.1.1.

\section{1-3. - Dose rate from outer walls}

The photon flux density from a vertical plane rectangular source with the concentration $Q$, length $2 L$, and height $h$ is calculated as follows with reference to figure 3 :

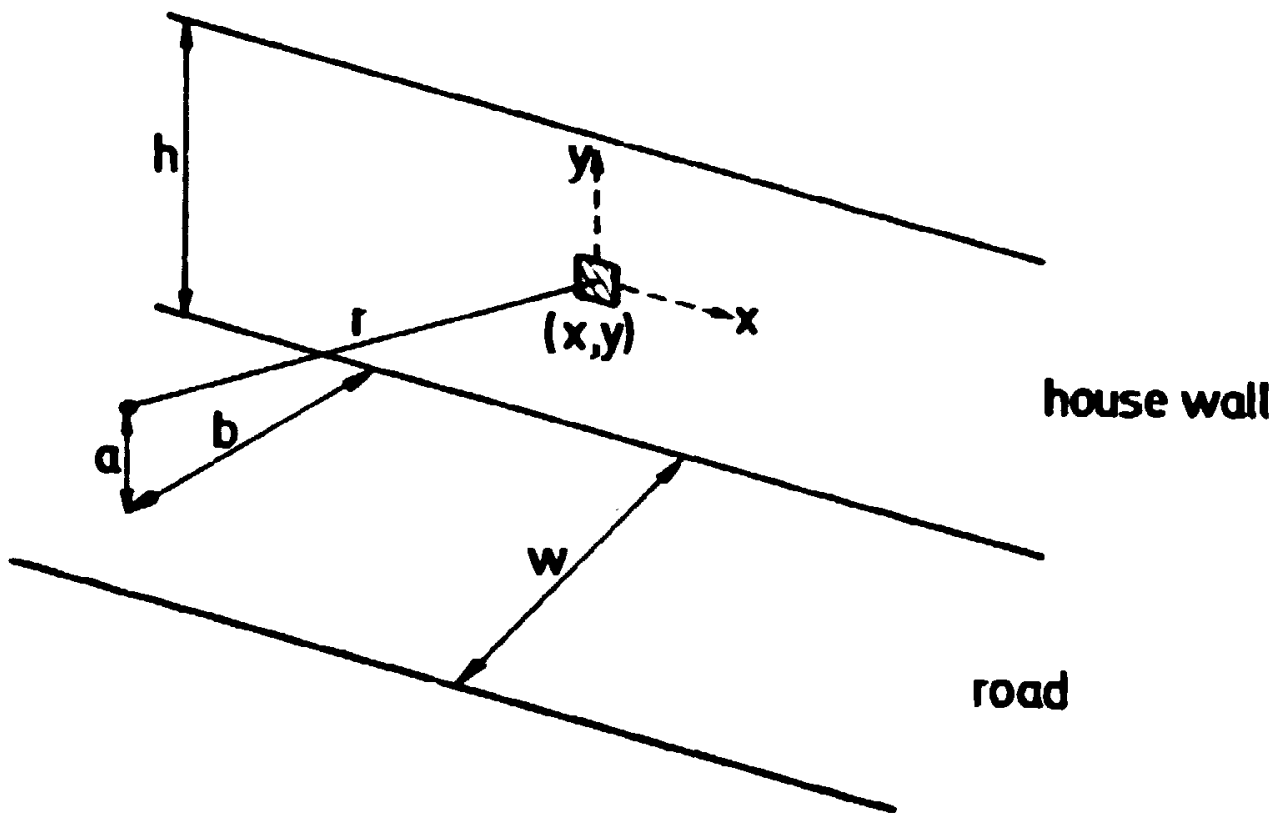

fig. 3. Geometry for calculating the dose rate from a wall of length $2 \mathrm{~L}$ and height $h$.

The photon flux density at the detector point from the infinitesimal wall element is:

$$
\begin{aligned}
& d \varphi_{\text {wall }}=\frac{Q \cdot d x \cdot d y}{4 \pi r^{2}} B\left(\mu_{a i r} \cdot r\right) \cdot e^{-\mu} \text { air } r \\
& r^{2}=x^{2}+y^{2}+b^{2}
\end{aligned}
$$

The total flux density is then: 


$$
\text { twall }=\int_{-L}^{L} \int_{-a}^{h-a} \frac{Q}{1-r^{2}} \cdot B\left(\mu_{a i r} \cdot x\right) \cdot e^{-\mu_{a i r} x} d y \cdot d x
$$

The ourdoor dose rate at a distance a above ground and b from one wall is:

$$
\dot{D}_{\text {out }, w}=\frac{Q}{4 \pi}\left(\frac{\nu_{e n}}{\rho}\right) \underset{\text { air }}{E} \cdot \int_{-L}^{L} \int_{-a}^{h-a} \frac{B\left(\psi_{a i r} r\right)}{r^{2}} \cdot e^{-\psi_{a i r} x} d y \cdot d x
$$

The outdoor dose rate from the walls on both side of a road is calculated from Eq. 13 as a sum of two terms with distances $b$ and w-b from the walls, respectively.

\subsection{Indoor dose rate}

2.2.1._Dose rate_fron a finite_ruad surface

The indoor photon flux density from deposited activity on the ground can be calculated from the following general equation:

$$
=\frac{Q}{4 \pi} \int_{A} \frac{B\left(\sum \mu R\right)}{r^{2}} e^{-\sum \mu R} d A
$$

where $I_{\mu R}$ is the sum of all the mean free paths in the building materials and air through which the photons will penetrate during travel from source to detector point. $A$ is the area with the activity concentration $Q$.

The geometry used for calculating of the indoor flux density in a multistorey building from surrounding ground surfaces is shown in Pigs. 4, 5, and 6 .

It appears from the figures that the road is divided in subareas from which the photons will penetrate various numbers of walls and floors. Only one inner wall parallel to the front 
wall is used in this model (in a single-family house two inner walls parallel to the long and short outer wall, respectively.

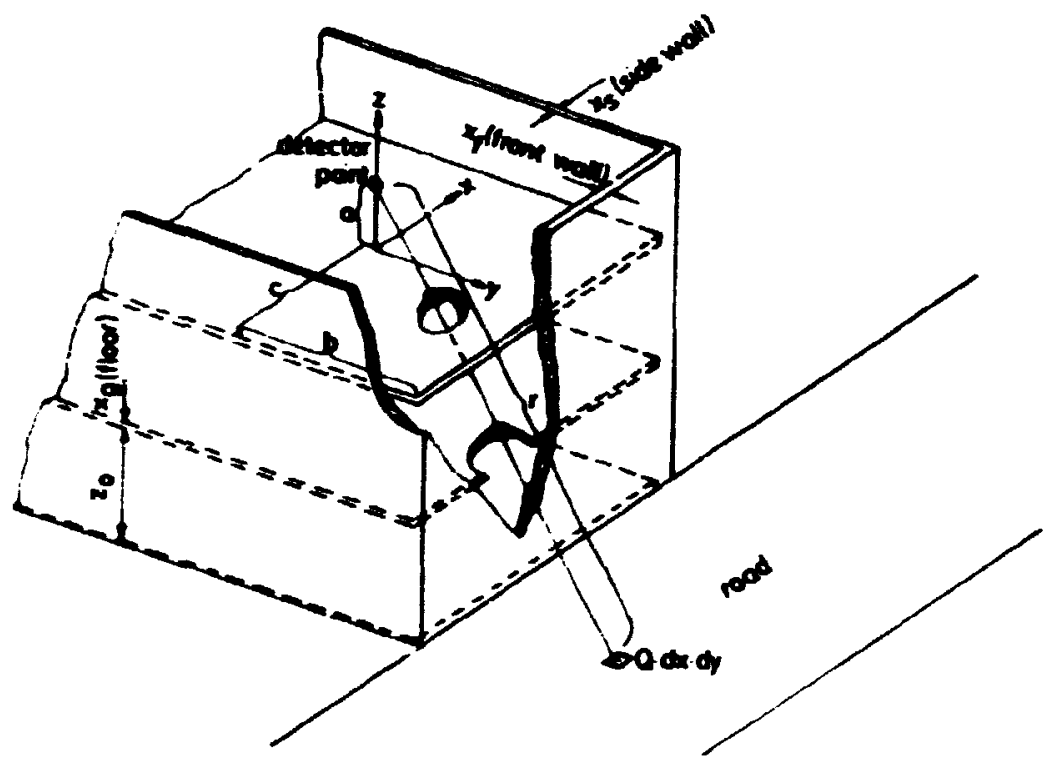

Fig. 4. Geometry for a multistorey building.

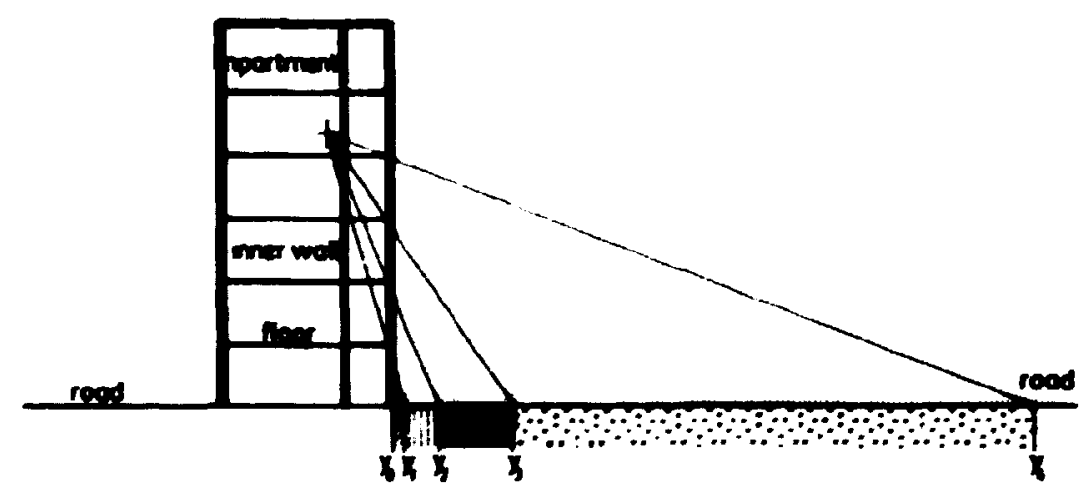

Pig. 5. Vertical cross section with sub-division of the road along a multistorey building. 


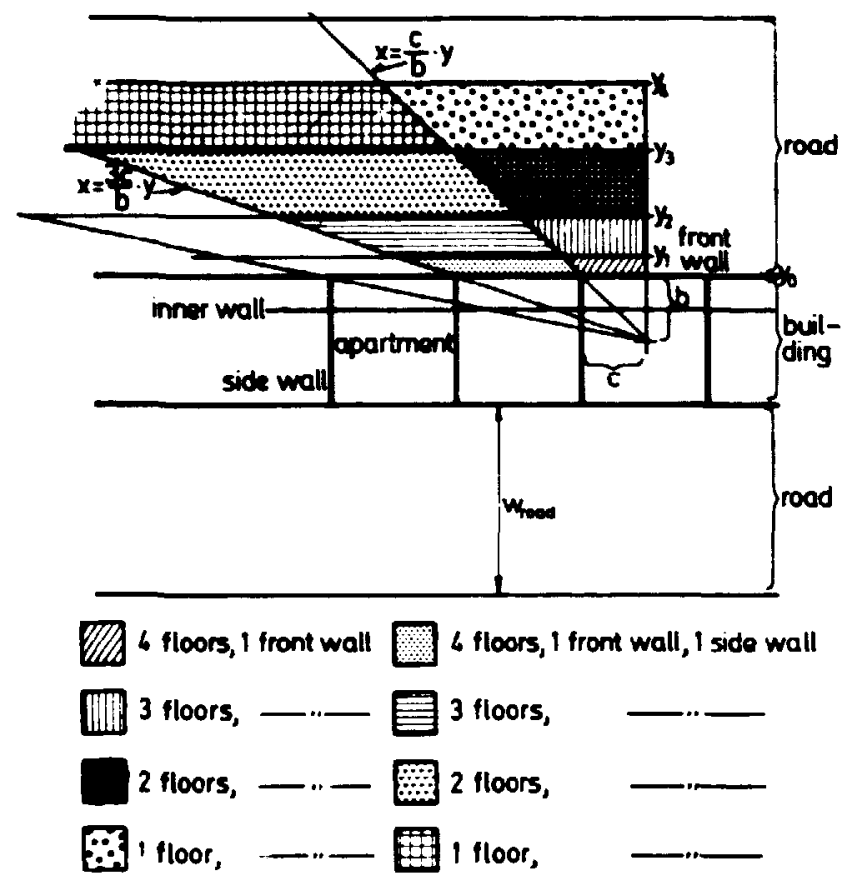

Fig. 6. Horizontal cross sertion with sub-division of the road along a multistorey building.

The photon flux density at the n'th floor is given by:

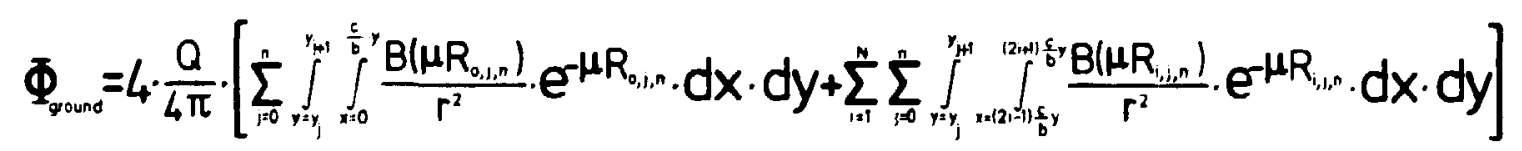

where

$$
r^{2}=x^{2}+y^{2}+\left(n\left(z_{0}+x_{g}\right)+a\right)^{2}
$$

$$
\begin{aligned}
\mu R_{1, j, n} & =\mu\left[(n-j) \cdot x_{g} \cdot \frac{r}{n\left(z_{o}+x_{g}\right)+a}+\left(x_{f}+x_{i n}\right) \frac{r}{y}+i x_{s} \frac{r}{x}\right] \\
& +\mu_{a i r} r ; \quad j=0,1,2 \ldots \ldots . n \\
y_{j}=b & \frac{n\left(z_{0}+x_{g}\right)+a}{(n-j)\left(z_{o}+x_{g}\right)+a}, \quad j=1,2, \ldots \ldots . . .
\end{aligned}
$$




$$
y_{0}=b+x_{f}
$$

$Y_{n+1}=Y_{0}+w_{\text {road }}$

$\mathbf{N}=$ number of neighbour apartments on each side of the apartment in question.

The second term in the bracket in Eq. 15 accounts for the radiation that - beyond the front wall - penetrates an increasing number of side walls between the apartments (up to $\mathrm{N}$ ).

The factor 4 in Eq. 15 takes inco account the presence of a road on each side of the building, and that the $x$-integration due to symmetry has been limited to start at zero.

In the computer program the total flux density for a multistorey building is calculated from Eq. 15 as:

$\Phi_{\text {ground }}=0.5 \cdot \Phi_{\text {ground }}\left(x_{\text {in }}=0 ;+0.5 \cdot\right.$ ground $\left(x_{\text {in }}\right)$

because only one inner wall $\left(x_{i n}\right)$ parallel to the outer wajl is considered. For single family houst the cotal flux density is calculated from Eq. 15 without the second term in the bracket as:

$$
\begin{aligned}
\Phi_{\text {ground }}=0.25 & {\left[\left(\Phi_{\text {ground }}\left(x_{\text {in }}=0\right)+\Phi_{\text {ground }}\left(x_{i n}\right)\right)_{b c}\right.} \\
+ & \left.\left(\Phi_{\text {ground }}\left(x_{i n}=0\right)+\Phi_{\text {round }}\left(x_{i n}\right)\right)_{c b}\right]
\end{aligned}
$$

The indices $b c$ and $c b$ indicate that $b$ and $c$ are interchanged (see Fig. 4) accounting for the dose rate contribution from the activity on the ground areas in front of the two long and the two short walls of the house, respectively.

The indoor dose rate from the ground is then given by:

$$
\dot{D}_{i n, g}=\Phi_{g r o u n d} \cdot\left(\frac{\mu_{e n}}{\rho}\right)_{a i r} \cdot E
$$


To account for the poor shielding of windows an effective thickness $x_{f}$ of the outer wall is used. The value is determined from the fraction $p$ of the outer wall area that is covered by windows and the gamma radiation transmission factors $T$ for the window and the outer wall:

$$
\begin{aligned}
T\left(\bar{x}_{f}\right) & =p \cdot T\left(x_{\text {window }}\right)+(1-p) \cdot T\left(x_{f}\right) \\
& \simeq p+(1-p) \cdot T\left(x_{f}\right)
\end{aligned}
$$

This method is considered somewhat conservative as it assumes that a fraction $p$ of the indoor residence time is spent at the windows (see sect. 4.1).

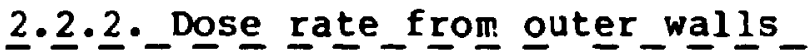

The indoor photon flux density from activity deposited on outer wall surfaces can be calculated from the basic Eq. 14. The geometry used for the calculation is shown at Fig. 7 .

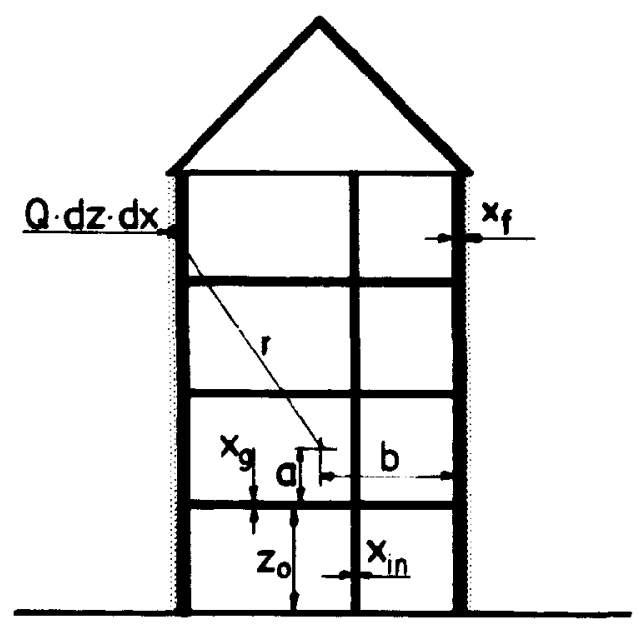

Fig. 7. Vertical cross section for calculation of indoor dose rate from depositea activity on outer walls.

The photon flux density at the n'th floor from activity deposited on the outer walls on a building with $p$ storeys is given by: 


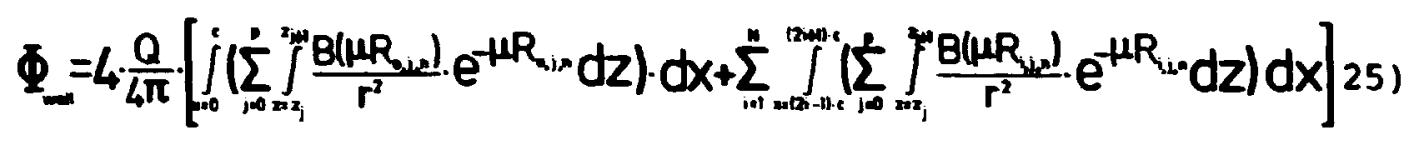

where

$$
\begin{aligned}
r^{2} & =\left(n\left(z_{0}+x_{g}\right)+a-z\right)^{2}+\left(b+x_{f}\right)^{2}+x^{2} \\
z_{j}=j \cdot\left(z_{o}+x_{g}\right) & \\
\mu R_{i, j, n}= & \mu\left[|n-j| \cdot x_{g} \cdot \frac{r}{\left|n\left(z_{o}+x_{g}\right)+a-z\right|}+\left(x_{f}+x_{i n}\right) \cdot \frac{r}{b+x_{f}}\right. \\
& \left.+i \cdot x_{s} \frac{r}{x}\right]+\mu_{a i r} \cdot r
\end{aligned}
$$

The second term in the bracket in Eq. 25 accounts for the radiation that penetrates both the outer wall and an increasing number of side walls between the apartments (up to $N$ ).

The factor 4 in Eq. 25 takes into account the presence of an outer-wall at each side of the building, and that the $x$-integration due to symmetry has been jimited to start at zero.

The total flux density for a multistorey building is calculated from Eq. 25 as:

$$
\Phi_{\text {wall }}=0.5 \cdot \Phi_{\text {wall }}\left(x_{\text {in }}=0\right)+0.5 \cdot \Phi_{\text {wa11 }}\left(x_{\text {in }}\right)
$$

as only one inner wall $\left(x_{i n}\right)$ parallel to the outer wall is considered. For single family houses the total flux density is calculated from Eq. 25 without the second term in the bracket as :

$$
\begin{aligned}
\Phi_{\text {wall }}=0.25 & {\left[\left(\Phi_{\text {wall }}\left(x_{i n}=0\right)+\Phi_{\text {wall }}\left(x_{\text {in }}\right)\right)_{b c}\right.} \\
& \left.+\left(\Phi_{\text {wal }}\left(X_{\text {in }}=0\right)+\Phi_{\text {wall }}\left(x_{\text {in }}\right)\right)_{c b}\right]
\end{aligned}
$$


The indices bc and $c b$ indicate that $b$ and $c$ are interchanged (see Fig. 4) accounting for the dose rate contribution from the activity on the two long and the two short walls of the house, respectively.

The indoor dose rate from the outer walls is then given by:

$$
\dot{D}_{\text {in,w }}=\Phi_{\text {wall }} \cdot\left(\frac{\text { Hen }}{\rho}\right)_{\text {air }} \cdot \mathbf{E}
$$

\section{$\underline{2} \cdot \underline{2} \cdot \underline{3} \cdot$-Dose $\underline{\text { rate_f }}$ from a $\underline{\text { roof }}$}

The indoor Elux density from activity deposited on the roof can be calculated from the basic Eq. 14. The geometry used is shown in Fig. 8. It appears from the figure that the tilted roof has been set equal to a flat roof at a distance aroof from the ceiling of the upper apartment.

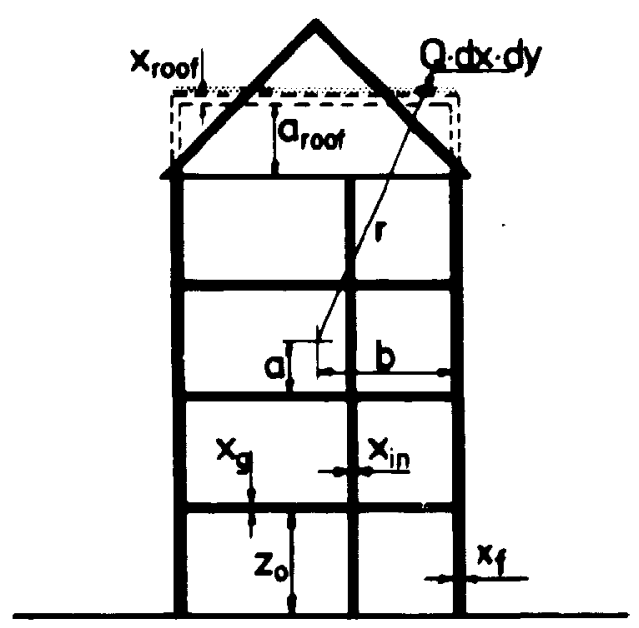

Fig. 8. Vertical cross section for calculation of indoor dose rate from deposited activity on a roof.

The photon flux density at the $n^{\prime}$ th floor arising from deposited activity on the roof on a bujlding with $p$ storeys is given by: 


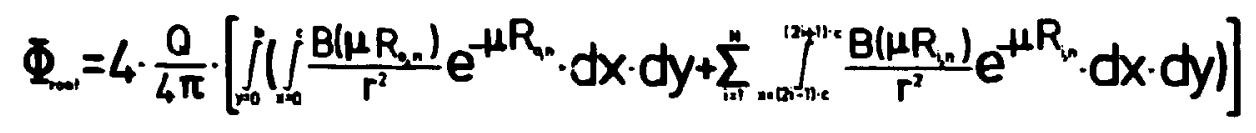

where

$$
\begin{aligned}
r^{2}= & \left((p-n) \cdot\left(z_{o}+x_{g}\right)+z_{o}+a_{\text {roof }}+x_{\text {roof }}-a\right)^{2}+x^{2}+y^{2} \\
\mu R_{i, n}= & \mu\left[\left((p-n) \cdot x_{g}+x_{\text {roof }}\right) \cdot \frac{r}{(p-n) \cdot\left(z_{o}+x_{g}\right)+z_{o}+a_{\text {roof }}+x_{\text {roof }}-a}\right. \\
& \left.+x_{i n} \cdot \frac{r}{\sqrt{x^{2}+y^{2}}}+i x_{s} \frac{r}{x}\right]+\mu_{a i r} \cdot r
\end{aligned}
$$

The secord term in the bracket in Eq. 32 accounts for the radiation that penetrates both the roof and an increasing number of side walls between the apartments (up to $\mathrm{N}$ ).

The factor 4 in Eq. 32 takes into account that both the $x$ - and $y$-integration due to symmetry has been limited to start at zero.

The total flux density for multistorey and single-family buildings is calculated from Eq. 32 as:

$$
\left.\Phi_{\text {roof }}=0.5 \cdot \Phi_{\text {roof }}\left(x_{\text {in }}=0\right)+0.5^{\circ} \phi_{\text {roof }} ! x_{\text {in }}\right)
$$

as only one inner wall parallel to the outer wall is considered. The indoor dose rate from the roof is then given by:

$$
\dot{\mathrm{D}}_{\text {in,r }}=\phi_{\text {roof }} \cdot\left(\frac{\text { Hen }_{\rho}}{{ }_{\text {air }}} \cdot \mathrm{E}\right.
$$




\section{SHIELDING FACTORS FOR BUILDINGS}

\subsection{Definition of the shielding factor}

The shielding factor is defined as

$$
S=\frac{\dot{D}}{\dot{D}_{\text {ref }}}
$$

where $\dot{D}$ is the actual dose rate and $\dot{D}_{\text {ref }}$ a reference dose rate.

In this study the reference dose rate is chosen as the dose rate 1 meter above an infinite, smooth surface source (cf. Sect. 2. 1.1).

\subsection{Outdoor shielding factor}

A person staying out of doors in a contaminated urban area will receive radiation doses from the contaminated roads as well as from house walls. However, the buildings will act both as radiation source and shield for radiation from distant parts of the ground surface, and consequently the dose rate in urban areas will be less than that from an infinite surface source with the same activity concentration. The outdoor shielding factor is given as:

$$
S_{\text {out }}=\frac{\dot{D}_{\text {out ,g }}+\dot{\mathrm{D}}_{\text {out ,b }}+\dot{\mathrm{D}}_{\text {out }, w-b}}{\dot{\mathrm{D}}_{\text {ref }}}
$$

The dose rate from the ground $\dot{D}_{\text {out, }} g$ is calculated by Eq. 9 in Sect. 2.1.2, and the dose rate from the buildings at each side of the road $\dot{E}_{\text {out }, b}$ and $\dot{D}_{\text {out, }} w-b$ are calculated from Eq. 13 in Sect. 2.1.3. The outcoor radiation at ground level from activity deposited on roofs is neglected, because a substantial part of it will be absorbed in the building materials. 


\subsection{Shielding factor for a single residence}

The radiation doses at indoor residence in a multistore; building or a single family house will come from contaminated roads, gardens, house walls, and roofs. Neighbouring houses will act both as a shield for the radiation from distant parts of the ground surface, and as a radiation source. In this study, the radiation from neighbouring houses is neglected, as their contribution to the total indoor dose rate is insignificant as far as Danish building/road geometries are concerned.

The indoor shielding factor is given by:

$$
s_{i n}=\frac{\dot{D}_{i n, g}+\dot{D}_{i n, w}+\dot{D}_{i n, r}}{\dot{D}_{\text {ref }}}
$$

The indoor dose rates are calculated from Equations 23, 31 and 36 , respectively.

\subsection{Weighted shielding factor}

For a given distribution of residences, a weighted shielding factor is defined as:

$$
\bar{s}_{i n}=\sum_{i} p_{i} \cdot s_{i n, i}
$$

where $\mathrm{p}_{i}$ is the fraction of a given residence type (single family, 3rd storey in a 7 storey house etc.) having the shielding factor $s_{i n, i}$.

The weighted shielding factor expresses the ratio between the average individual dose rate for the area under consideration when all individuals remain indoors, and the dose rate for the same area when all individuals remain out of doors on an infinite surface with the same activity concentration.

With an equal distribution of people in the given distribution of residences, the weighted shielding factor also ex- 
presses the ratio between the collective dose rate from indoor residence, and the collective dose rate to the population from outdoor residence on an infinite surface source.

\subsection{Time-averaged shielding factor}

The weighted shielding factor $\overline{\mathbf{s}}_{\text {in }}$ is a static shielding factor for a given geographic area as far as residence in homes is concerned. However, it is necessary to consider different 10cations during a week, i.e. out of doors, working place, transport, and at home. This dynamic shielding factor or time-averaged shielding factor is defined as:

$$
\bar{s}_{t}=\sum_{i}\left(\frac{t_{i}}{168}\right) \cdot s_{i}
$$

where $t_{i}$ is the number of hours in a week during which an individual is located at a place having the shielding factor $s_{i}$ (which can either be a weighted shielding factor or a single shielding factor).

The above is correct only when the different shielding factors $\mathbf{s}_{i}$ refer to areas with the same activity concentration as the reference area. This implies that the population of a certain district is employed within this district. of course, this is a simplification but the error is somewhat counterbalanced in the summing of the collective dose for many municipalities.

Furthermore, it should be noted that both the weighted and the time-averaged shielding factors should be used only to calculate collective doses and average individual doses for the evaluation of stochastic health effects, but not for calculating individual doses for the assessment of non-stochastic health effects. For this purpose, the area under consideration should be divided in shielding classes and a timemaveraged shielding factor calculated for each. 


\subsection{Calculated shielding factors for Danish houses}

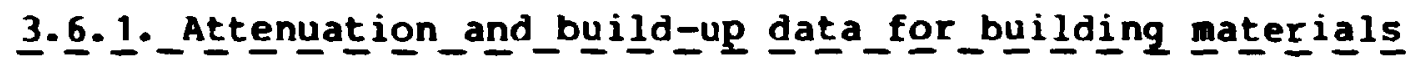

The attenuation coefficient for the building materials depends on the elemental composition and the microscopic photon cross section for these elements. The linear attenuation coefficient is calculated from:

$$
u=\rho \cdot \sum_{i} \frac{6.023 \cdot 10^{23}}{A_{i}} \cdot \frac{w_{i}}{100} \cdot \sigma_{i}
$$

where $w_{i}$ is the weight per cent, $\sigma_{i}$ the total microscopic cross section (excl. the coherent cross section) in barn/atom, and $A_{i}$ the atomic weight for element no. $i$ in building material of density $\rho$.

Typical Danish bricks have the following composition (Je78) as shown in Table 1 .

\begin{tabular}{|c|c|c|}
\hline \multicolumn{2}{|c|}{ Element composition for Danish bricks, $w_{i}$ 8 } \\
\hline Element & Red bricks & Yellow bricks \\
\hline \multirow{2}{*}{$\mathrm{Si}$} & 48 & 42 \\
$\mathrm{Al}$ & 21 & 17 \\
$\mathrm{Fe}$ & 21 & 12 \\
$\mathrm{Ca}$ & 6 & 7 \\
$\mathrm{Mg}$ & - & 17 \\
$\mathrm{~K}$ & 1.6 & 2.9 \\
$\mathrm{Na}$ & 3.6 & 3.3 \\
\hline
\end{tabular}

Table 1. Weight percentages of elements in typical Danish bricks.

The linear attenuation coefficients for red and yellow bricks have been calculated with values of $\sigma_{i}$ from sto 67 . Table 2 shows the attenuation coefficients used in this study, calculated as average values for red and yellow bricks. 


\begin{tabular}{|c|c|}
\hline \multicolumn{2}{|c|}{ Linear attenuation coefficient for Danish bricks } \\
\hline Photon energy (MeV) & $\begin{array}{c}\text { Linear attenuation } \\
\text { coefficient, } \mu\left(\mathrm{cm}^{-1}\right)\end{array}$ \\
\hline 0.1 & 0.2996 \\
0.2 & 0.2126 \\
0.3 & 0.1820 \\
0.5 & 0.1483 \\
1.0 & 0.1082 \\
1.5 & 0.08809 \\
2.0 & 0.07605 \\
\hline
\end{tabular}

Table 2. Linear attenuation coefficients for typical Danish bricks.

Values of $\mu_{a i r}$ and ( $\left.\mu_{e n} / \rho\right)$ air are also taken from sto 67.

The density for bricks is assumed to be $1.7 \mathrm{~g} \cdot \mathrm{cm}^{-3}$, and in all the calculations the building materials have been set equivalent to a brick thickness $x_{\text {eq }}$ defined as:

$$
x_{e q}=\frac{x_{m}}{\rho}
$$

where $x_{m}$ is the mass-thickness in $g^{\circ} \mathrm{cm}^{-2}$ and $\rho$ is the density of bricks.

Dose build-up factors for water have been used, because the difference between build-up factors for light elements is of minor importance.

Capo's bivariant formulae ( $\mathrm{Ca} 58$ ) for the calculation of build-up factors have been used.

$$
B(E, \mu x)=\sum_{i=0}^{3} \sum_{j=0}^{4} c_{i j}\lfloor\mu x]^{i} \cdot E^{-j}
$$




$$
\begin{aligned}
& =\sum_{i=0}^{3} a_{i}(E) \cdot[u x]^{i} \\
B_{i}(E) & =\sum_{j=0}^{1} c_{i j} \cdot E^{-j}
\end{aligned}
$$

\begin{tabular}{|c|c|c|c|c|c|c|c|c|}
\hline \multicolumn{9}{|c|}{ Polynominal Coefficients, $c_{i j}$} \\
\hline$j / i$ & 0 & & 1 & & 2 & & 3 & \\
\hline 0 & 1.01094 & $E+0$ & 1.16772 & $E-1$ & -7.65869 & $E-3$ & 1.67068 & $E-4$ \\
\hline 1 & -6.00394 & $\mathbf{E}-2$ & 2.32125 & E+o & -1.79023 & $\mathbf{E}-2$ & 5.69295 & $E-4$ \\
\hline 2 & 7.20778 & E-2 & -2.12801 & $E+0$ & 2.41735 & $E-1$ & -7.96332 & E-3 \\
\hline 3 & -3.01498 & $E-2$ & 7.67783 & $E-1$ & -4.34443 & $E-2$ & 7.23758 & $E-3$ \\
\hline 4 & 3.94733 & $E-3$ & -9.08139 & E-2 & -1.34203 & $E-2$ & -9.87237 & $E-4$ \\
\hline
\end{tabular}

The Capo coefficients are shown in Table 3.

Table 3. Capo cuefficients for dose build-up factors for water.

The coefficients are defined for the following intervals:

$$
\begin{gathered}
0.255 \mathrm{MeV} \leq \mathrm{E} \leq 10 \mathrm{MeV} \\
0 \leq \mu \mathrm{X} \leq 20
\end{gathered}
$$

For photon energies below $0.255 \mathrm{MeV}$ the coefficients for 0.255 MeV are used.

3.6.

Based on data from the Danish Building Research Institute shielding factors have been calculated for typical Danish houses. These shielding factors have been used as the basis for the parameter study in sect. 4. The data used in the calculations are given in Table 4. 


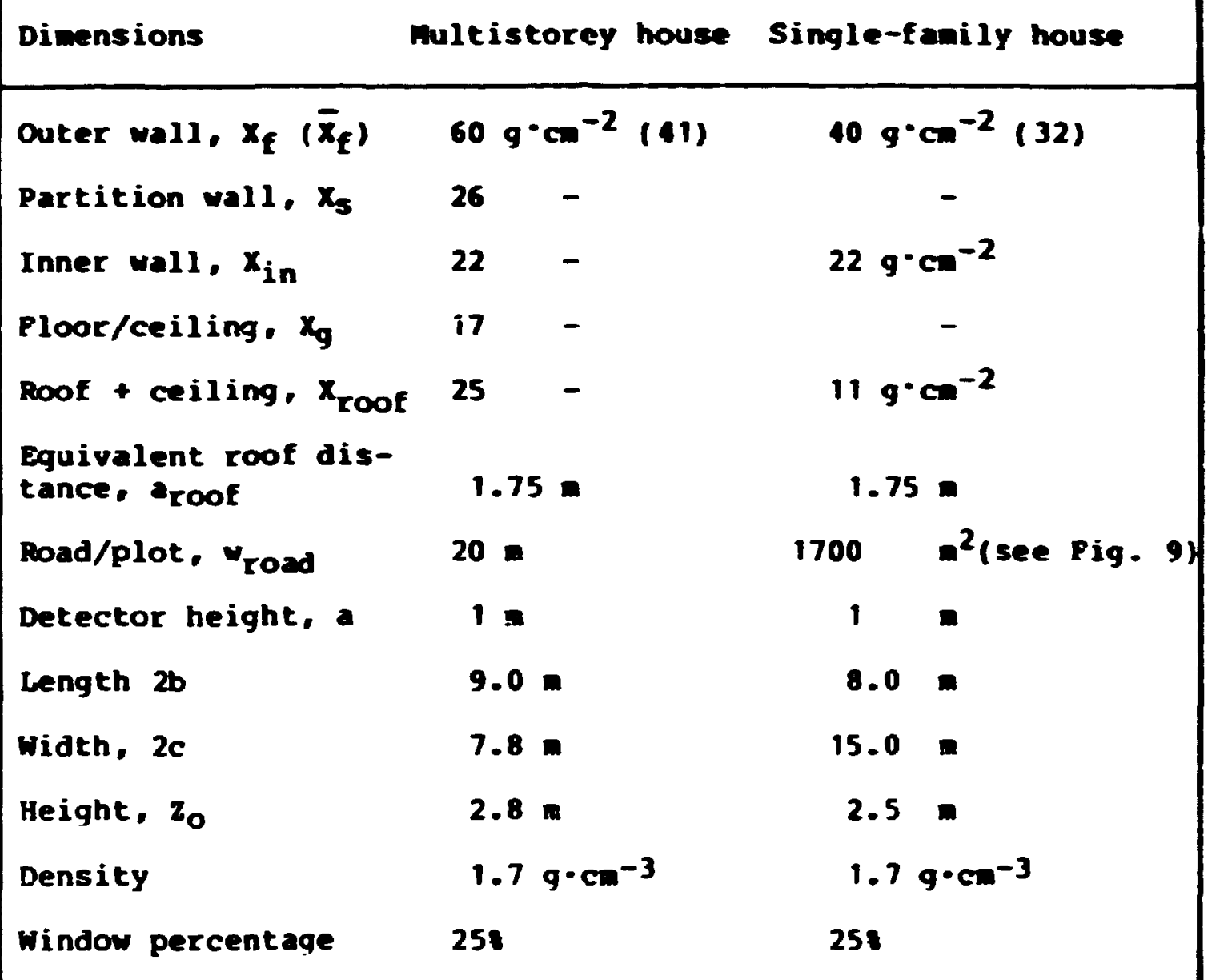

Table 4. Data for single-family and multistorey houses.

In Pigures 9 and 10 the ground areas included in the dose rate calculations are shown for single-family and multistorey buildings, respectively. The calculatione have been performed by numerical integration. A simpson formula has been used, and the numerical accuracy in the calculations is 0.18 . 


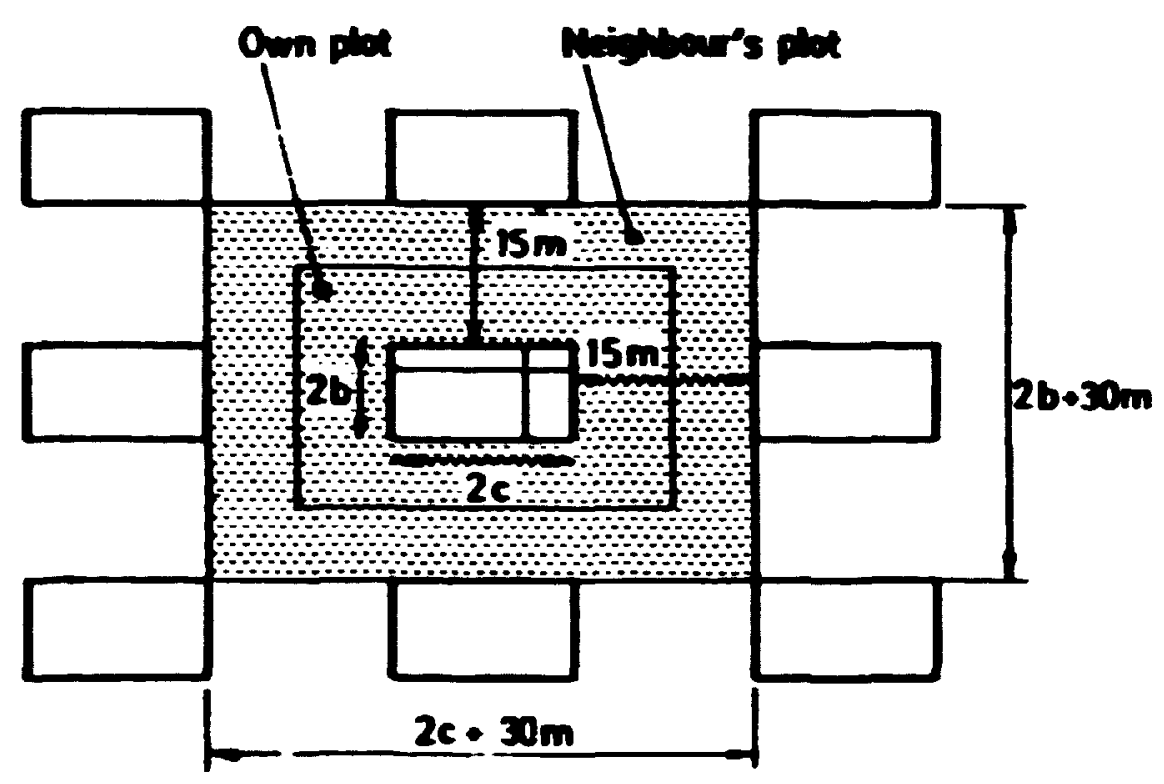

pig. 9. Geonetry for single-family house areas.

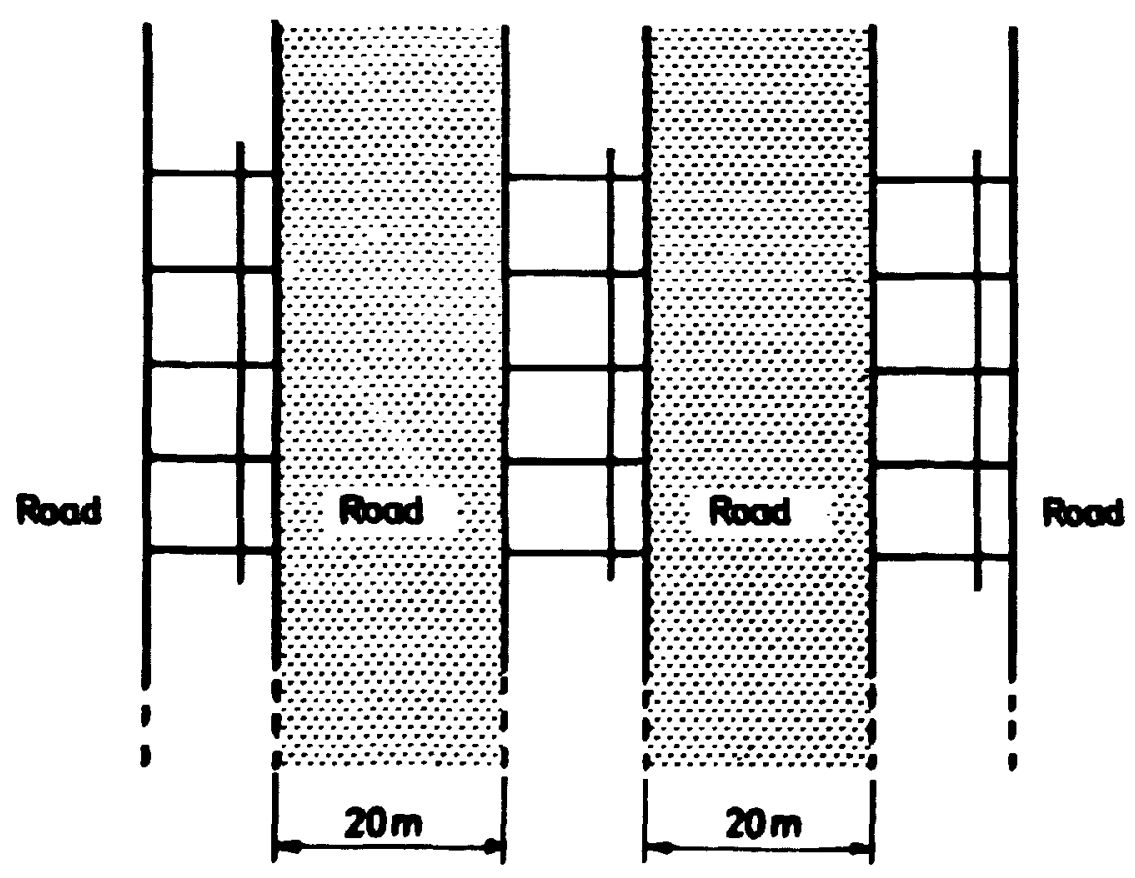

Pig. 10. Geometry for multistorey house areas. 
Table 5 shows the calculated shielding factors for $0.5 \mathrm{MeV}$ photons assuming a uniform surface activity distribution on all surfaces for multistoreyed buildings, and a ten-times larger surface activity on the ground than on the roof and outer walls for single-family houses. The reason for the latter is that experiments indicate that the deposition velocity for smooth surfaces like houses and asphalt roads is more than one magnitude less than for grass surfaces (Ro81).

For single-family houses, the shielding factor is found to be approximately 0.07, corresponding to a reduction factor of 15 of the outdoor dose rate from an infinite surface source having the same deposition as the gardens surrounding the houses. For multistorey buildings, the largest reduction is found in the highest buildings, and here for the apartments on their middle floors. In this case the outdoor dose rate from an infinite surface source having the same deposition as on houses and roads is reduced by a factor of up to 100 (shielding factor 0.01 ). For smaller buildings, the shielding factor for apartments on the middle floors is about 0.02, whereas in all cases the apartments on the bottom and top floors give poorest protection, corresponding to a shielding factor of 0.03-0.04.

\begin{tabular}{|c|c|c|c|c|c|c|c|c|c|c|c|}
\hline $\begin{array}{c}\text { Storey } \\
\text { no. }\end{array}$ & Single & 1-st. & 2-st. & 3-st & 4-st. & 5-st. & 6-st. & 7-st. & 8-st & 9-st. & 10 -st. \\
\hline 0 & .065 & .036 & .032 & .031 & .031 & .031 & .031 & .031 & .031 & .031 & .031 \\
\hline 1 & & .039 & .019 & .015 & .014 & .014 & .014 & .014 & .014 & .014 & .014 \\
\hline 2 & & & .034 & .015 & .011 & .010 & .0098 & .0098 & .0098 & .0098 & .0098 \\
\hline 3 & & & & .034 & 015 & .011 & .0099 & .0097 & .0097 & .0097 & .0097 \\
\hline 4 & & & & & .034 & .014 & .011 & .0097 & .0096 & .0095 & .0095 \\
\hline 5 & & & & & & .034 & .014 & .010 & .0096 & .0094 & .0094 \\
\hline 6 & & & & & & & .034 & .014 & .010 & .0095 & .0093 \\
\hline 7 & & & & & & & & .034 & .014 & .010 & .0094 \\
\hline 8 & & & & & & & & & .034 & .014 & .010 \\
\hline 9 & & & & & & & & & & .034 & .014 \\
\hline 10 & & & & & & & & & & & .034 \\
\hline
\end{tabular}

Table 5. Calculated shielding factors for Danish houses. 
Experiments have been carried out in the USA to determine the shielding factor for houses, primarily for free-standing, onestoreyed or two-storeyed houses ( $\mathrm{Au} 59, \mathrm{Bu} 66, \mathrm{Bu} 70$ ). In order to simulate a homogeneously distributed surface source on the grour.d and on the roof, use was made of a tube system placed around the house and on the roof; a relatively strong radioactive source was pumped through the tubing at a constant rate. Integrating dosimeters were set up in the different rooms in the house to measure the dose from the rotating source. These experiments were intended to clarify the shielding effect of typical American houses in the event of fallout from nuclear weapons.

For houses built of brick or concrete blocks, reduction factors in the 5-10 range were found away from doors and windows in the outer rooms. Reduction factors of 10 to 20 were found in inner rooms ( $\mathrm{Bu} 66$ ). Taking into account the differences between building traditions and standards in the USA and Denmark, there is reasonably good agreement between the experimental results and the calculations used in the present study. In the Reactor Safety study (WASH-1400) a reduction factor of 5 was used (Bu 75).

The distribution of different houses in the city of Cupenhagen (given by the Danish Department of Statistics), and the assumption that the surface activity concentration is the same on single-family houses, multistorey houses and ground in urban areas, and furthermore a factor of 10 higher on the ground in areas with single-family houses will give the following weighted shielding $\overline{\mathrm{s}}_{\text {in }}$ :

$$
\bar{s}_{\text {in }}=0.006
$$

This means that the average individual indoor dose rate for the municipality of Copenhagen is 170 times less than the dose rate one meter above an infinite surface having the same activity concentration as the gardens surrounding single-family houses. 
With the same assumptions concerning surface concentration a time-averaged shielding factor can be calculated from:

$$
\begin{aligned}
& \quad \bar{s}_{t}=\left\{\frac{t_{i}}{168} \cdot s_{i}\right. \\
& =168^{-1}\left[p _ { s } \cdot \left(t_{i n} \cdot s_{\text {in }}+t\right.\right. \text { out } \\
& \left.\cdot s_{\text {out }}+t_{\text {trans }} \cdot s_{\text {trans }}+t_{\text {work }} \cdot s_{\text {work }}\right)_{\text {single }} \\
& \left.+\left(1-p_{s}\right) \cdot\left(t_{\text {in }} \cdot s_{\text {in }}+t_{\text {out }} \cdot s_{\text {out }}+t_{\text {trans }} \cdot s_{\text {trans }}+t_{\text {work }} \cdot s_{\text {work }}\right)_{\text {urban }}\right]
\end{aligned}
$$

where $t_{\text {in, }} t_{\text {out }} t_{\text {trans }}$ and $t_{\text {work }}$ are the times during a week spent indoors, outdoors, in transport vehicles, and at work and $S_{\text {in. }} S_{\text {out, }} S_{\text {trans, }}$ and $S_{\text {work }}$ are the corresponding shielding factors with reference to the dose rate one meter above an infinite surface with the same concentration as for single family house gardens. The ratio of single-family houses to the total number of residences in the Copenhagen area, $p_{s}$, is 0.0592 . In the calculation of $\bar{s}_{t}$ it is assumed that a represen.tative shielding factor for work/ school, $S_{\text {work, has appro- }}$ ximately the same value as for a first floor apartment in a 3-storeyed house, based on the consideration that factories, public offices, hospitals, colleges, schools, etc.. are normally larger buildings with thick walls. Sout has been calculated

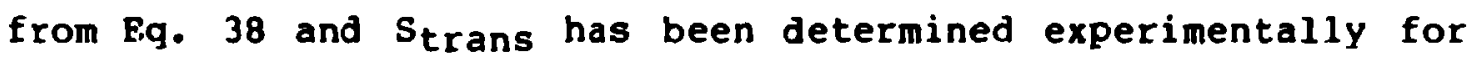
transport vehicles ( La 82). Table 6 shows the values for the parameters used to calculate $\bar{s}_{t}$.

\begin{tabular}{|lccl|}
\hline Location & Time distribution & $\begin{array}{l}\text { Single-family } \\
\text { house area }\end{array}$ & $\begin{array}{l}\text { Urban } \\
\text { area }\end{array}$ \\
\hline Outdoors & 6.28 & 0.6 & 0.06 \\
Transport & 5.08 & 0.25 & 0.030 \\
Work/school & 23.88 & 0.0015 & 0.0015 \\
Home & 65.08 & 0.065 & $\left\{p_{i} S_{i}=0.0023\right.$ \\
\hline
\end{tabular}

Table 6. Shielding factors for different locations. 
With these values the time-averaged shielding factor for the municipality of Copenhagen have been calculated to the following value:

$$
\bar{s}_{t}=0.012
$$

meaning that the average individual dose rate over a week for persons that live and work in the Copenhagen area is 80 times less than the dose rate one meter above an infinite surface with the same activity concentration as the single-family house gardens.

\section{PARAMETER STUDY}

\subsection{Window areas}

When staying indoor near windows the shielding for gamma radiation from activity deposited on outer walls and ground surfaces is negligible. This is in the model achieved by defining an effective (fictive) front-wall thickness that has the same transmission factor for gamma radiation as a combination of the transmission factors for the front wall and windows. Is $p$ the fraction of indoor residence time spent at windows, $x_{f}$ and $x_{w i n d o w}$ the thickness of front wall and windows, then an effective front-wall thickness $\bar{x}_{f}$ can be calculated from:

$$
\begin{aligned}
T\left(\bar{x}_{f}\right) & =p \cdot T\left(X_{w i n d o w}\right)+(1-p) \cdot T\left(X_{f}\right) \\
& =p+(1-p) \cdot T\left(X_{f}\right)
\end{aligned}
$$

where $T$ is the transmission factor defined as the product of the dose build up factor and the attenuation factor:

$$
B\left(\bar{X}_{f}\right) \cdot e^{-\mu \bar{X}_{f}}=p+(1-p) \cdot B\left(X_{f}\right) \cdot e^{-\mu X_{f}}
$$

In this study the fraction of indoor residence time $p$ spent at windows is set equal to the fraction of the outer walls that 
is covered by windows. This reflects the fact that the dose rate at any detector point in the house contains both a "window" component and an "away from window" component. However, the method is considered somewhat conservative for at least two reasons. Firstly, it is assumed that the activity deposited on the windows has the same concentration as that on the walls. It will probably be significantly lower. Secondly, the "window" component from activity deposited on the ground will only from a fraction of the ground area surrounding the building. In the model the total ground area will be included.

Fig. 11 shows the effective front wall thickness $\bar{x}_{f}$ as a function of the front-wall thickness $x_{f}$ for a number of p-values between 0.2 and 0.6 , and valid for a photon energy of $0.5 \mathrm{MeV}$.

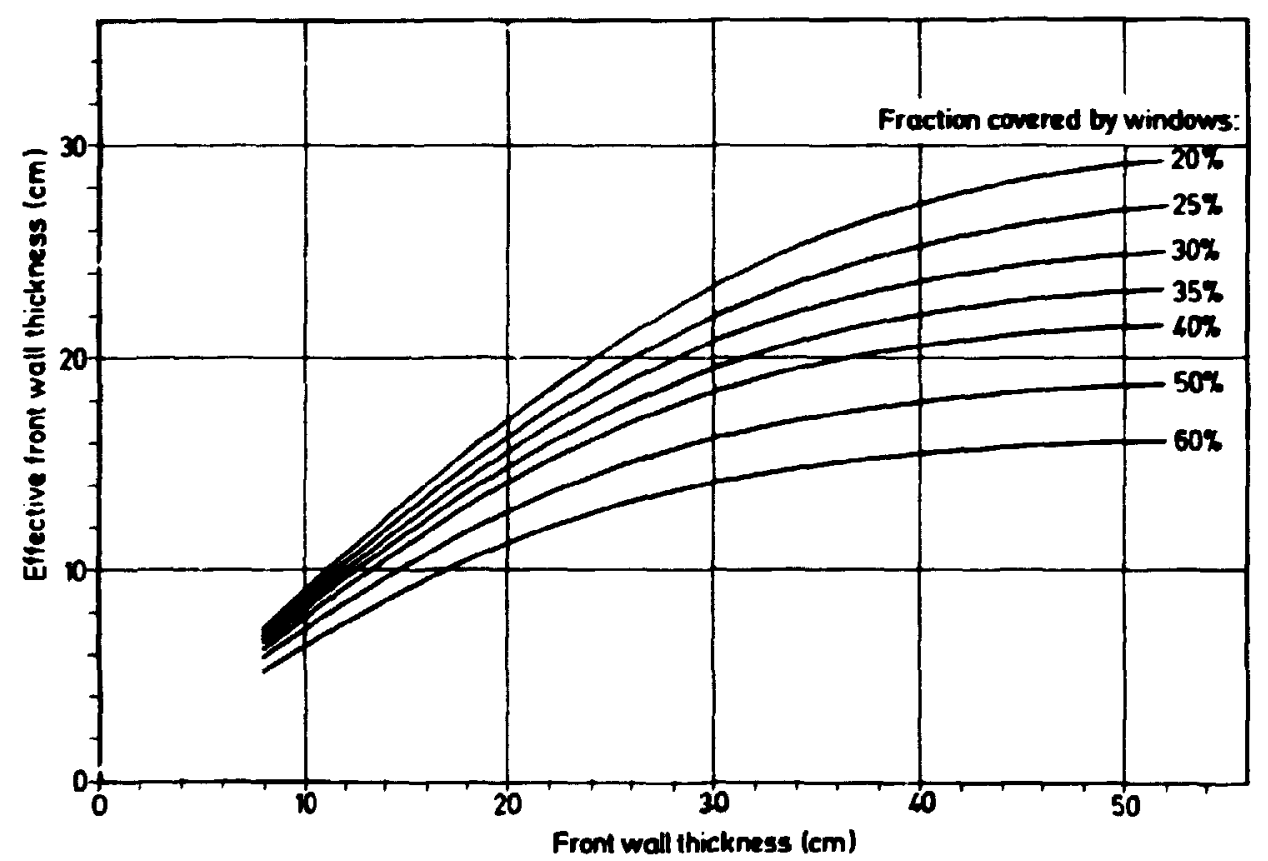

Fig. 11. Effective front-wall thickness for $0.5 \mathrm{MeV}$ photons.

The shielding factors shown in Table 5 in Sect. 3.6 .2 have been calculated for a window fraction of 258 of the outer wall area. Fig. 12 shows the corresponding shielding factors for different window fractions ranging from $108-508$. 
$-31-$

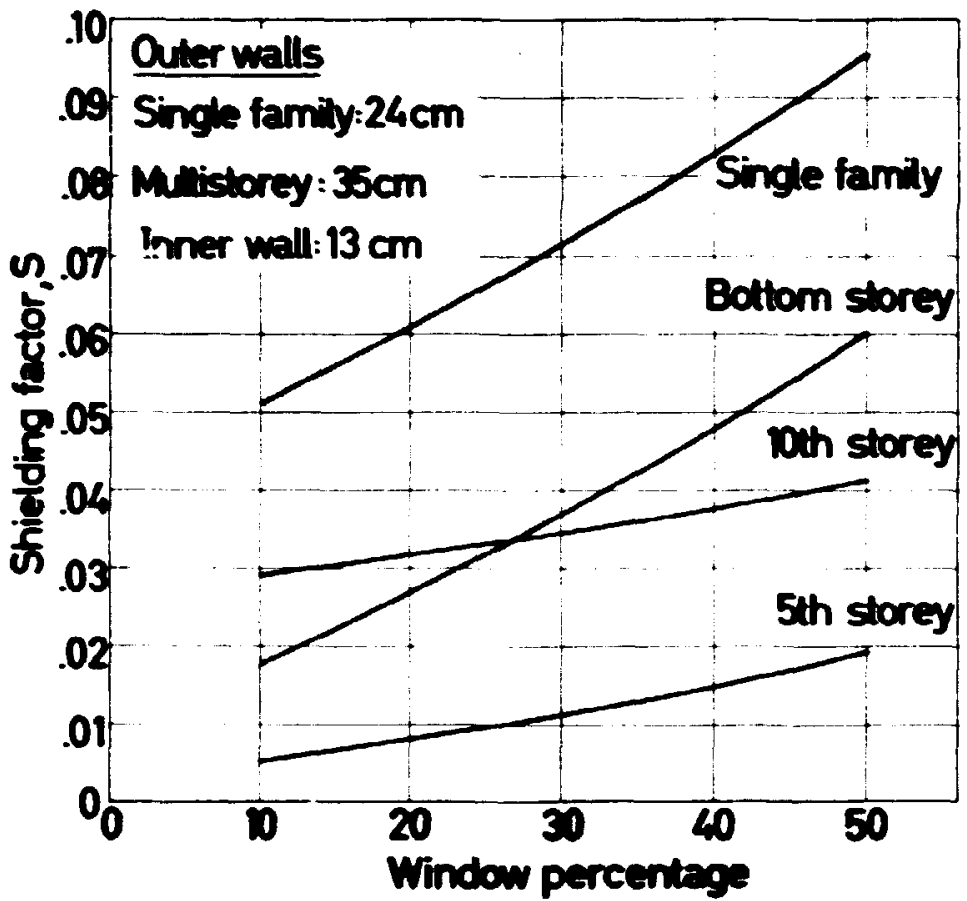

Fig. 12. Shielding factors as a function of window fraction for a single family and a 10-story house.

For single-family houses ca. 908 of the indoor dose rate originates from activity deposited on the ground and 38 from activity on the wall. This is not surprising as it is assumed that the activity concentration on the ground is ten times larger than on the building surfaces. When the window percentage is increased from 108 to 508 the dose rate from the outer wall (plus windows) and ground is increased by a factor of 2 for the given building dimension. Therefore, the shielding factor will increase with nearly a factor of 2 as shown in Fig. 12.

For multistorey buildings with ten storeys 998 of the indoor dose rate in the bottom floor originates from activity deposited on the ground and outer wall 1718 from road and 288 from outer wall). The dose rate from these two sources will both increase by a factor of 3.4 for the given building dimension when the window fraction is increased from 108 to 508 and, consequently, so will the shielding factor.

At the fifth floor ca. 978 of the indoor dose rate originates from activity on the outer wall. When the window fraction is increased from 108 to 508 the dose rate from the wall will increase with nearly a factor of 4. Therefore, the shielding factor will increase nearly with the same factor. 
At the tenth floor the dose rate from the roof accounts for 60:-808 of the total dose rate, depending on the window fraction. The rest of the dose rate originates from the walls as the contribution from the road is negligible. Due to the relatively large contribution from the roof the total dose rate at the upper floor will be less dependent on the window fraction, even when the dose rate from the walls will increase with almost a factor of 4 if the window fraction is increased from 108 to 508 . As seen at Fig. 12 the shielding factor is increased only by approximately 408 .

\subsection{Outer-wall thickness}

When the outer-wall thickness is either increased or decreased the indoor dose rate will correspondingly increase or decrease. The shielding factors shown in Table 5 have been calculated for an outer-wall thickness of $35 \mathrm{~cm}$ for a multistoreyed building and $24 \mathrm{~cm}$ for a single-family house. Figure 13 shows the corresponding shielding factors for other values of outer wall thickness.

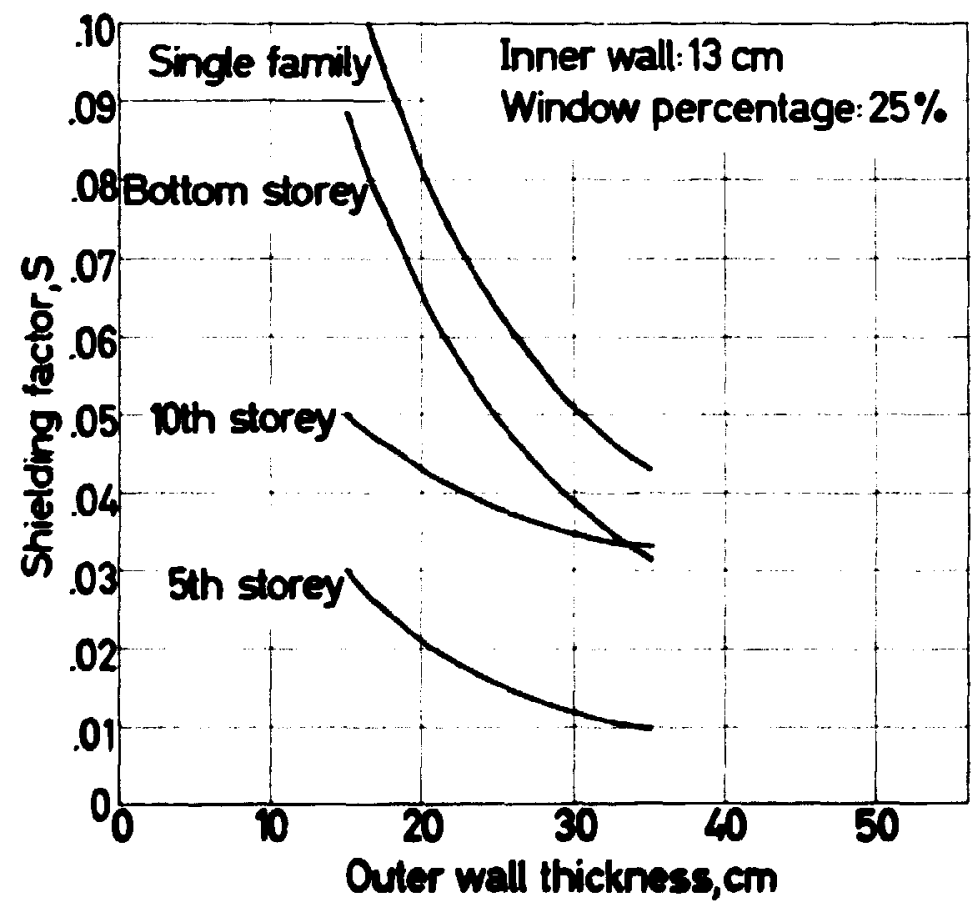

Fig. 13. Shielding factors as a function of outer-wall thickness for a single-family and a 10-storey house. 
If the outer-wall thickness for a single-family house is increased from $15 \mathrm{~cm}$ to $35 \mathrm{~cm}$, the indoor dose rate contribution from activity deposited on the walls and the ground will decrease with approximately a factor of 3 . The dose rate from the surrounding ground area will contribute with 808-908 of the total indoor dose in that interval, and the shielding factor will correspondingly decrease with a factor of approximately 2.5.

At the bottom floor in a multistorey building the indoor dose rate from activity deposited on ground and wall will decrease with approximately a factor 3 when the outer-wall thickness is increased from $15 \mathrm{~cm}$ to $35 \mathrm{~cm}$. Consequently, the shielding factor will decrease with the same factor, as 998 of the dose rate originates from ground and outer-wall.

At the fifth floor the dose rate is dominated by the radiation from activity deposited on the outer-wall, i.e. 95-978 of the total. This contribution will decrease with approximately a factor of 3 for the outer-wall interval mentioned. Therefore, the shielding factor will also decrease with a factor 3 .

At the tenth floor the dose rate from the roof accounts for 508 - 708 of the total dose rate, depending on the outer wall thickness in the interval $15-35 \mathrm{~cm}$. The remaining dose rate originates from the outer wall with a corresponding contribution of 508-308. The shielding factor will therefore be less dependent of the outer-wall thickness, and for the given building dimensions approximately a factor of 1.5 .

\subsection{Inner-wall thickness}

When the inner-wall thickness is increased the indoor dose rate will decrease and vice versa. When the inner-wall thickness is increased from 0 to $25 \mathrm{~cm}$, contribution to the indoor dose rate from activity deposited on the ground, outer-walls, and roof will all decrease with nearly a factor of 2, for both single family and multistoreyed houses. Therefore, the shielding factors will also decrease with approximately a factor of 2 as shown at Fig. 14. 


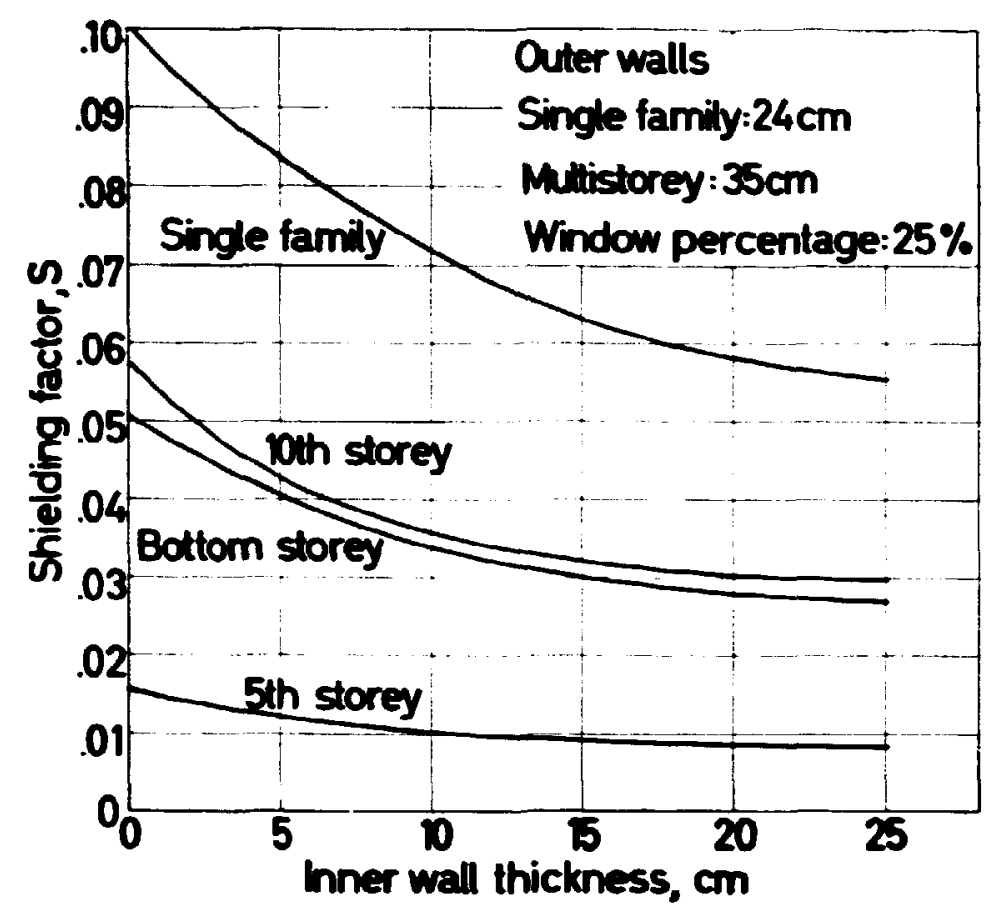

Fig. 14. Shielding factors as a function of inner-wall thickness for a single-family and a 10-storey house.

\subsection{Road and ground width}

The dose rate contribution from activity deposited on ground surfaces will - as far as multistorey buildings are concerned be the dominant contributor to the total indoor dose rate in the lower-placed apartments. In the higher-placed apartments the contribution of the ground surface source will fall due to increased distance and increased front-wall shielding. For single-family houses the ground surface will be far the most dominant source. In the shielding factors shown in Table 5 the indoor dose rate contribution from the ground surfar is 758, 38 and 08 of the total dose rate at the botton floor, fifth floor, and tenth floor, respectively, in a 10-storey building. For a single family house the corresponding figure is ca. $90 \%$. Therefore, when the ground and road surface width is increased (see Figs. 9 and 10 ) the shielding factors for a single-family house and lower-placed apartments will be the most affected, whereas for the higher-placed apartments the shielding factors will be nearly independent of changes of road width. 


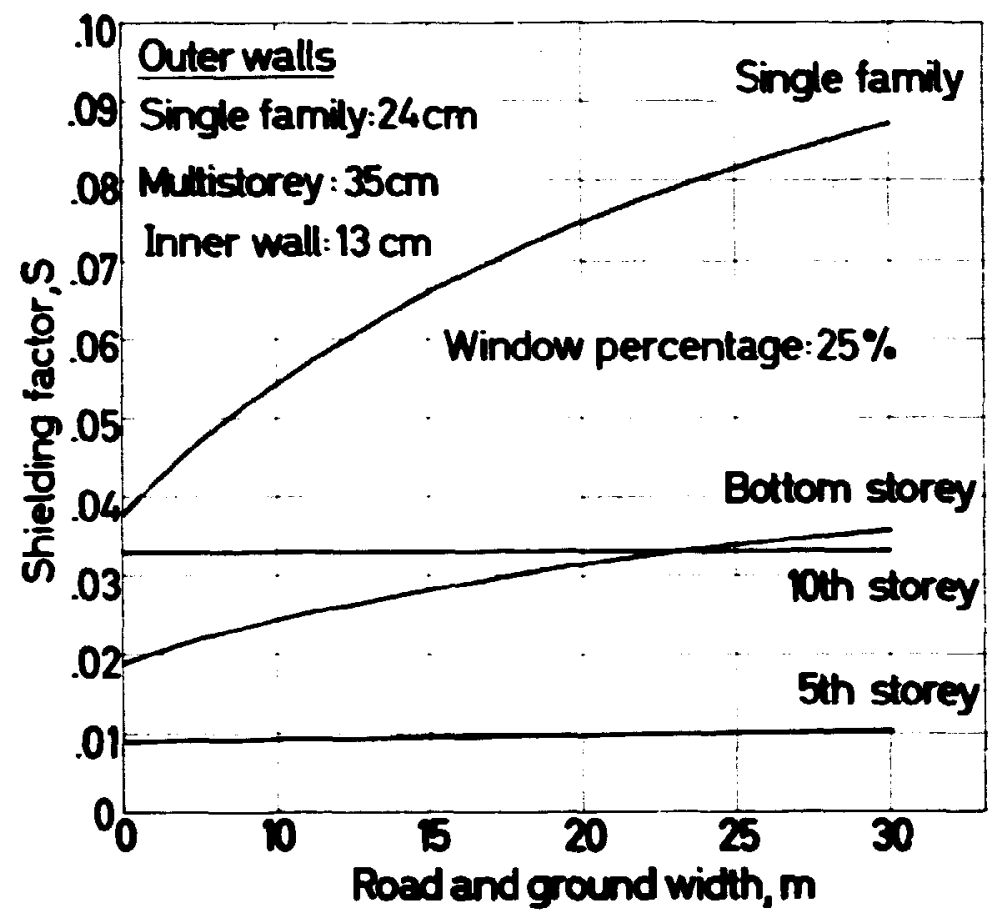

Fig. 15. Shielding factors as a function of road and ground width for a single-family and a 10-storey house.

It is seen from Fig. 15 that the botton storey and single-family shielding factors is increased by 258-358 when the ground and road width is increased by af factor of 2 of the values shown in Figs. 9 and 10. Purthermore, it appears that the shielding factor for a single-family house and at the bottom storey will decrease with approximately a factor of 2 if the ground and road width is decreased from 30 meters to 0 .

\subsection{Decontamination of surfaces}

The model for calculation of the shielding factors use inde pendent surface activity concentrations on the ground, walls, and roof. Therefore, the indoor dose rate reduction after a given decontamination effort can be found. Calling the shielding factor for the original deposition $s_{O}$, and the shielding factor calculated for the reduced concentration $5_{r e d}$, the indoor dose rate reduction from decontamination will be equal to $s_{0} / s_{\text {red. }}$ 
Fig. 16 shows the effect on the shielding factor for a single family house by decontamination of the different surfaces.

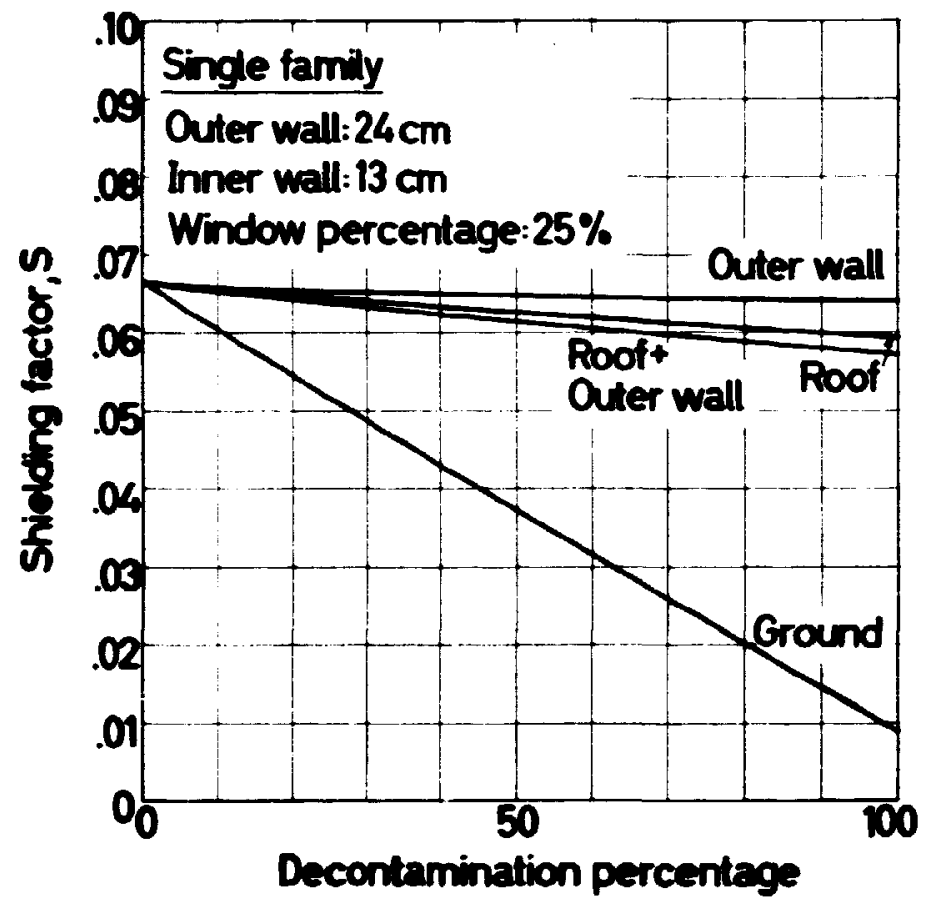

Fig. 16. Shielding factor for a single-family house as a function of the degree of decontamination.

It is seen that if the ground surface is decontaminated to 508 of the original deposition then the indoor dose rate is reduced by a factor of nearly 2. It also appears that even if the roof and outer walls are fully decontaminated the indoor dose rate reduction will be only $13 \%$. The reason for this is the assumption that the originally deposited activity concentration on ground surfaces is ten times higher than on outer-walls and roof (Ro 81 ).

For the lower apartment in a multistorey building the conditions will be slightly different as shown in Fig. 17. 


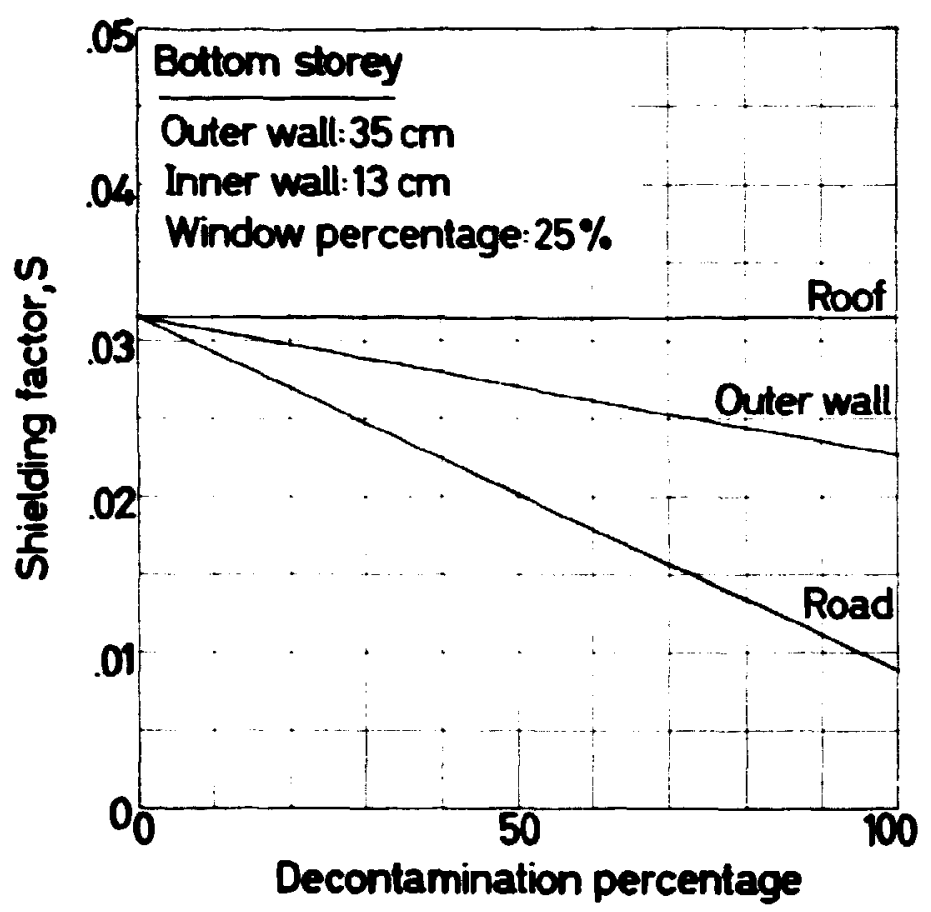

Fig. 17. Shielding factor for a 10-storey house as a function of the degree of decontamination.

Here the assumption is that the deposited activity concentration is the same on all surfaces including the road surface (Ro 81). Therefore, a decontamination of the outer-wall will have a larger influence than for a single-family house. A 50 o decontamination of road and outer-wall will reduce the indoor dose rate by a factor of exactly 2 because the roof component is negligible. If either the road or outer-walls are fully decontaminated the indoor dose rate will be reduced by 728 and $28 \%$, respectively.

For an apartment at the fifth floor in a 10-storey building the dose rate components from the roof and the road are negligible as shown in Fig. 18. This means that a given reduction of the outer-wall contamination will result in the same reduction of the indoor dose rate. 


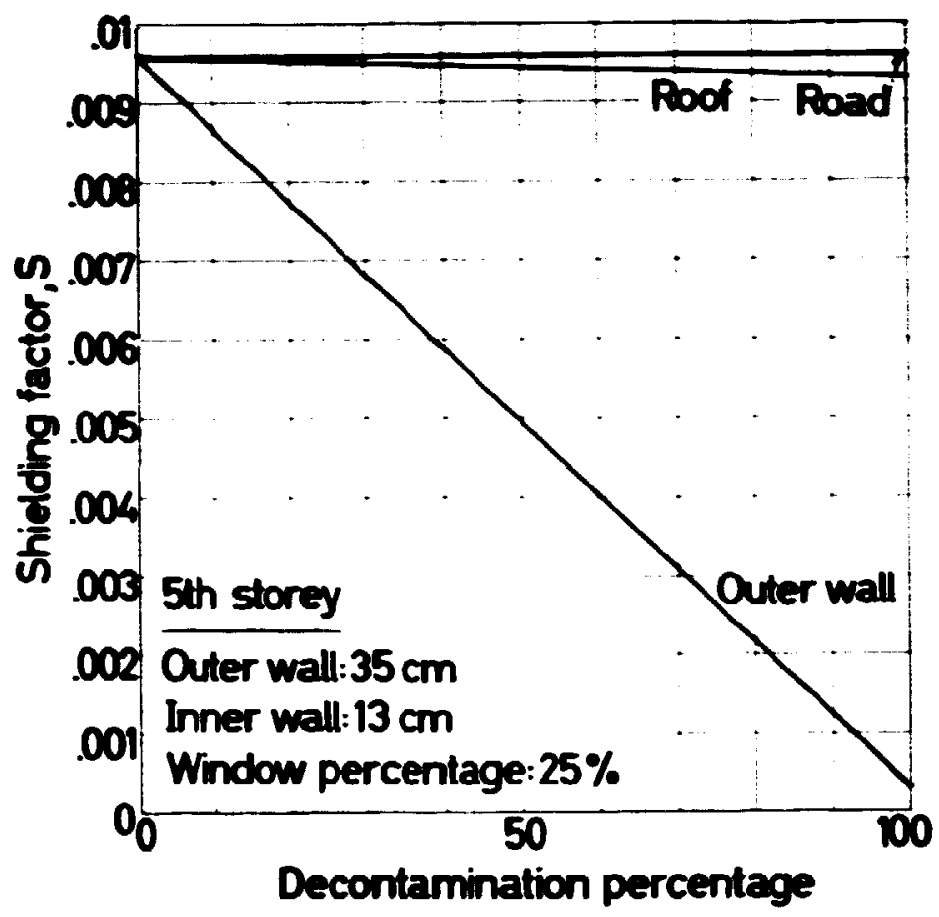

Fig. 18. Shielding factor for a 10-story house as a function of the degree of decontamination.

For the upper apartment the indoor dose rate is dominated by the activity deposited on the roof as shown in Fig. 19.

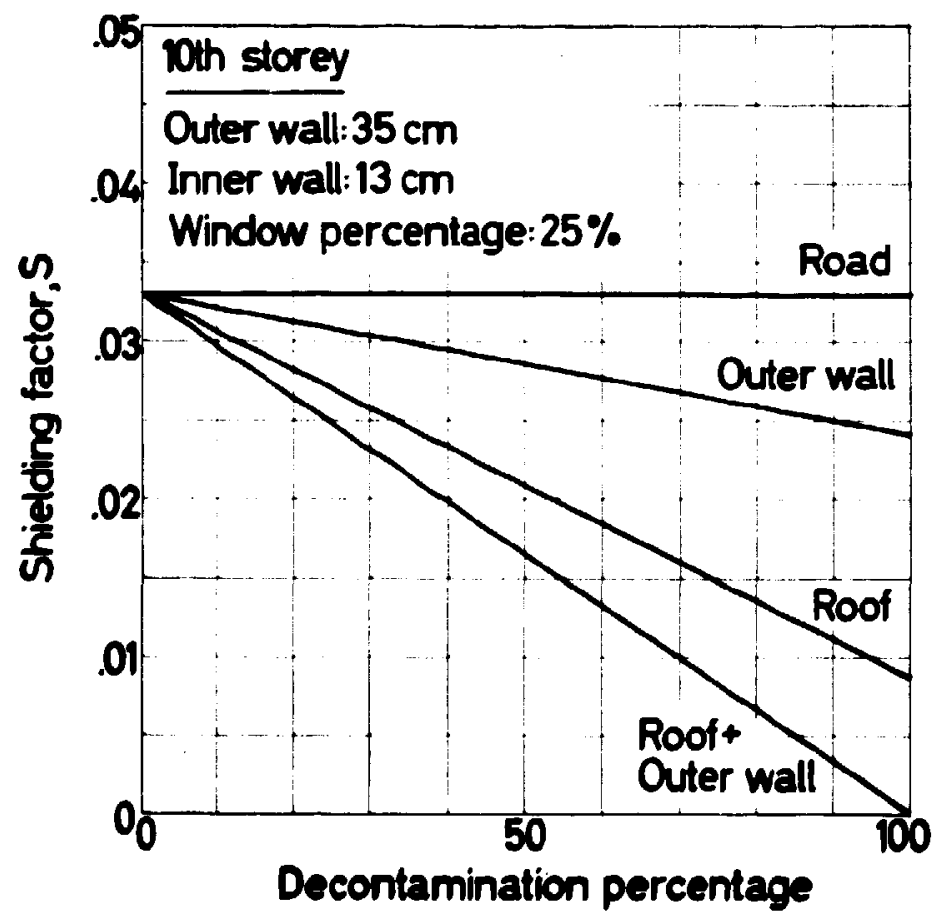

Fig. 19. Shielding factor for a 10-storey house as a function of the degree of decontamination. 
If the roof is decontaminated to 50 of the original depostion the indoor dose rate will be reduced by almost 40 . If either the roof or outer-walls are fully decontaninated the indoor dose rate will be reduced by 738 and 278 , respectively.

\subsection{Photon energy}

Photons of higher energy will be wore penetrating than photons of lower energy as seen from the linear attenuation coefficients shown in Table 2 in section 3.6.1. This inplies that indoor protection from radiation originating from deposited activity will be less for radionuclides with high photon energies than for those with low photon energies. The shielding factors will therefore increase with increasing photon energy. The relevant photon energies for radionuclides released by nuclear reactor accidents will be in the range from 0.1-1.5 Mev.

In Fig. 20 shielding factors are shown as a function photen energy for a single-family and a lo-storey house.

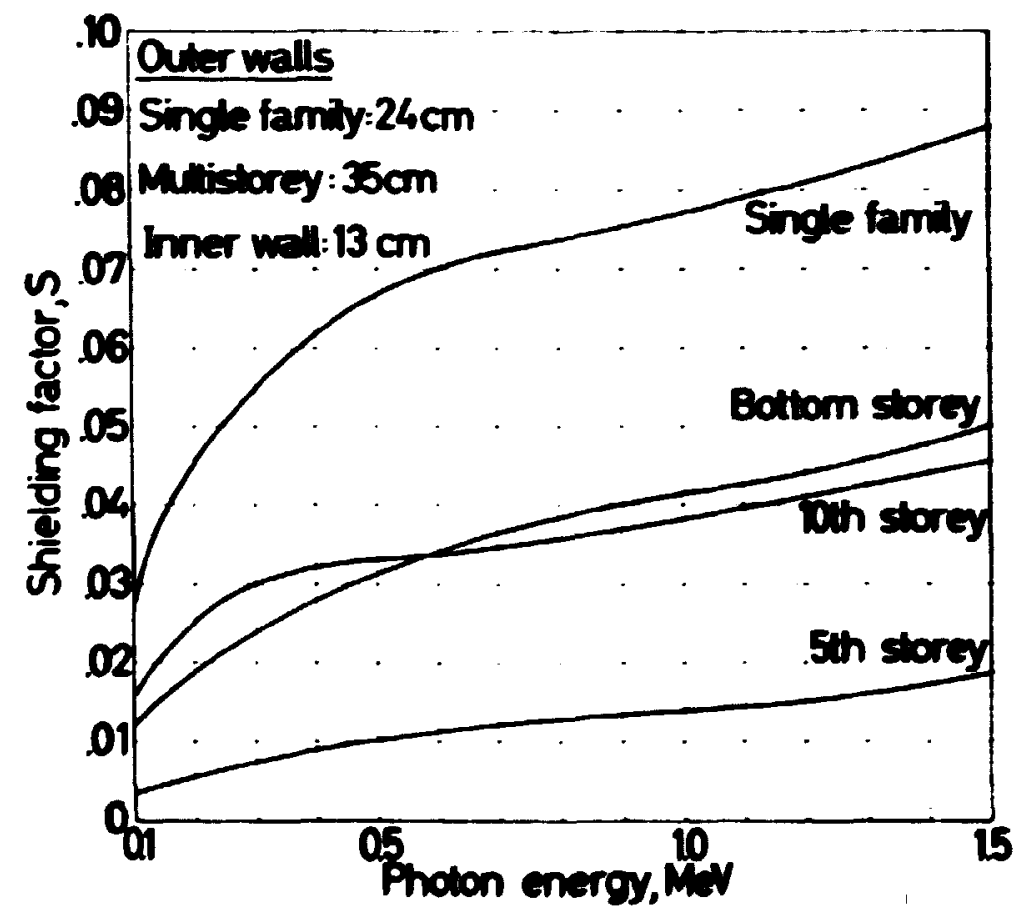

Fig. 20. Shielding factors as a function of photon energy for a single-family and a 10-storey house. 
It appears from the figure that the shielding factor is decreased by a factor of ca. 2, when the energy is decreased from $0.5 \mathrm{MeV}$ to $0.1 \mathrm{Mev}$ and increased by less than 50 pct, when the energy is increased from $0.5 \mathrm{MeV}$ to $1.5 \mathrm{MeV}$.

All the shielding factors shown in this report are calculated for a photon energy of $0.5 \mathrm{MeV}$. These values can be used with a fairly good approximation for radiaton from the longlived radionuclide $137 \mathrm{Cs}$, that has a photon energy of $0.66 \mathrm{MeV}$.

5. DISCUSSION OF UNCERTAINTIES

The uncertainty in the shielding factors calculated in this report is dependent on the uncertainties of the calculated dose rates from the different surface sources.

The fundamental calculation method used here is based on the socalled exponential point attenuation kernel that links the radiation flux density or other measurable quantity related to the flux density at a given detector point to the point-source strength. Attenuation resulting from geometrical spreading with increasing distance from the source as well as exponential attenuation and scattering of the photons are taken into consideration. The assumptions for the point kernel method are that both the source and shielding medium are isotropic, i.e. that the source radiaces uniformly in all directions and that the medium has the same attenuating properties in all directions. Besides, as far as scattering of photons is concerned it is assumed that both source and detector are located within an infinite homogeneous medium. For a given extended isotropic source the flux density is found by proper integration of the point kernel over the source extension (cf.Eq. 14 in sect. 2.2.i).

These assumption: are approximately fulfilled in the calculations of the reference dose rate (Eq. 4 Sec:. 2.1.1.) and the outdoor dose rate from finite surfaces (Eq. 9 and 13 in sect. 2.1 .2 and 2.1 .3 ) as verified by transport theory calcu- 
lations (Be72) and experimental measurements (La82). Therefore, the outdoor dose rate can be calculated with an accuracy of perhaps 10-208.

Otherwise, in the calculation of the indoor dose rate the assumption that the source and the detector should be located within an infinite medium is not quite fulfilled. As the dose build up factors have been derived for an infinite medium the indoor dose rate is overestimated in some cases when calculated from the point kernel method. The infinity assumption is nearly always fulfilled for the building walls in the plane perpendicular to the line from source to detector point. All the scattered photons in the wall will arise here from an arta within 2-3 mean free paths in the wall material around the primary photon direction, i.e. within a $10-40 \mathrm{~cm}$ radius, depending on the photon energy.

On the other hand, as the detector point inside an apartment is surrounded by air the infinity-assumption can never be fulfilled along the photon direction. Therefore, the photons that in an homogenous medium will be back-scattered from the semi-infinite medium behind the detector point will be lacking. However, it is believed that this is counterbalanced by the backscatter from inner walls, floor, and ceiling in the apartment. The back-scatter component that is contained in the dose build-up factor is of importance only for lower photon energies and can be neglected for energies above $1 \mathrm{MeV}(\mathrm{Ja68})$.

Another important source of uncertainty is the deposition velocities for the different surfaces as roads, gardens, walls, and roofs. In this report it is assumed that the deposition velocities to all other surfaces apart from gardens are equal, and in the case of gardens a factor of ten higher.

If, for instance, the deposition velocity to gardens was equal to that of all other surfaces, then the shielding factor for a single family-house would be doubled. Additionally, if the deposition velocity to outer walls was neglected as is done in several studies (Spe80, Bu75) then the shielding factor for the middle, floors in multistorey buildings would decrease 
by a factor of 30 . It is therefore very important when calculating realistic shielding factors that the ratio of the deposition velocities to the different surfaces are known.

Uncertainties will also depend on geometrical simplifications of the buildings and their surroundings and the data used in the calculations for attenuation, build-up, and energy absorption. However, it is believed that these uncertainties are of minor importance compared with those already mentioned.

As the uncertainty of the reference dose rate is small, the overall uncertainty of the calculated shielding factors is equal to the uncertainty of the calculated indoor dose rates. For given surface deposits, building dimensions, and surrounding area sizes it is believed that shielding factors for photon energies above $1 \mathrm{MeV}$ can be calculated with an accuracy of 20-308. For low photon energies the shielding factors can be somewhat overestimated by using build-up factors for an infinite homogeneous medium.

\section{CONCLUSION}

A computer model has been developed in order to calculate shielding factors for typical Danish houses for gamma radiation from fall-out radioactivity, and the results shown in this report have been used to calculate the consequences from hypothetical accidents at the Barsebäck nuclear power plant in Sweden (Gj82). The model can be used also for other specified house types, and it has in fact been used to calculate also the shielding factors for typical houses in the other Nordic countries (TV82).

An important feature of the model is that independent activity concentration is assumed at various surfaces. Therefore, it will be possible to analyse situations within risk studies where the deposition velocity to ground surfaces is different from that of house surfaces, as well as situations where de- 
contamination has changed the surface activity concentration on the different surfaces.

For gamma radiation from deposited 137 Cs a typical Danish singlefamily house will give a protection factor of $10-20$ relative to the outdoor dose rate one meter above an infinite surface source having the same deposition as on the surface of the surrounding gardens. Multistorey buildings will give corresponding protection factors of $25-100$ assuming that the infinite surface source has the same deposition as houses and roads.

For the Copenhagen area the time-averaged protection factor for $137 \mathrm{Cs}-\mathrm{radiation}$ has been calculated to 80 meaning that the average individual dose rate in the metropolitan area is 80 times less than the dose rate one meter above an infinite surface source with the same surface concentration as the single family house gardens in the city.

It should be emphasized however, that the time-averaged shielding factor should be used to calculate only the individual doses that could result in stochastic effects, and not the individual doses that could result in non-stochastic effects. For this purpose, the shielding factors in a given area should be divided in classes and a time-averaged shielding factor calculated for each. 
7. REFERENCES

Au 59 AUXIER, J.A. et al., 1959, Experimental evaluation of the radiation protection afforded by residential structures aqainst distributed sources", CEX-58.1, USAEC.

Be 72 BECK, H.L. et al., 1972, "In Situ Ge(Li) and NaI(T1) Gamma-Ray spectrometry", HASL-258, USAEC.

Bu 66 BURSON, 2.G., 1970, "Experimental radiation measurements in conventional structures. Part I: Radiation measurements in two two-storey and three one-storey typical residential structures before and after modification", CEX59.7B, USAEC.

Bu 70 BURSON, 2.G., 1970, "Experimental evaluation of the fallout radiation protection provided by structures in the contral point area of the Nevada Test site", CEX-69.5, USAEC.

Bu 75 BURSON, 2.3. and PROFIO, A.E., 1975, "structure Shielding from Cloud and Fallout Gamma Ray Sources for Assessing the Consequences of Reactor Accidents", EGG-1183-1670. Las Vegas, Nevada.

Ca 58 CAPO, M.A., 1958, "Polynomial Approximation of Gamma-Ray Buildup Factors for a Point Isotropic Source", Apex-510.

Gj 82 GJøRUP, H.L. et al., 1982, "Radioactive Contamination of Danish Territory after Core-melt Accidents at the Barsebäck Power Plant", Risø-R-462.

Ja 68 JAEGer, R.G. et al., 1968, "Engineering Compendium on Radiation Shielding: Shielding Fundamentals and Methods", Vol. I pp. 225-226, Springer-Verlag, Berlin Heidelberg New York. 
Je 78 JEnSEN, E.. 1978, "The typical Danish brick. A phase analytical study by X-rays", Kgl. Vet.- og Landbohøjskole. Arsskr. 1978: 53-63.

La 82 LAURIDSEN, B. and HEDEMANN JENSEN, P., 1982, "Shielding Factors for Vehicles to Gamma Radiation from Activity Deposited on Structures and Ground Surfaces", Risø-M-2339.

Ro 81 ROED. J.. 1981, "Surface deposition of airborne material released by a core-melt accident at a power reactor", Riso-M-2274 (in Danish).

Spe80 SPENCER, L.V., CHILTON, A.B. and EISENHAUER, C.M., 1980, - Structure Shielding Against fallout Gamma Rays from Nuclear Detonations". NBS Special Publication 570, U.S. Department of Commerce/National Bureau of Standards, Washington, D.C.

Sto67 STORM, E. and ISRAEL, H.I., 1967, "Photon Cross Sections from 0.001 to $100 \mathrm{MeV}$ for Elements 1 through 100", La3755, Los Alamos, New Mexico.

Tv 82 TVETEN, U., 1982, "Shielding Factors for Typical Houses in the Nordic Countries", IFE-KR-E-82-001-V, Institute for Energy Technology, Kjeller, Norway. 
SHIELDING FACTORS FOR GAMMA RADIATION FROM ACTIVITY DEPOSITED ON STRUCTURES AND GROUND SURFACES

Per Hedemann Jensen

Department or group

Health Physics

45 pages +6 tables +20 illustrations

Abstract

This report describes a computer model that calculates shielding factors for indoor residence in multistorey and single family houses for gamma radiation from activity deposited on roofs, outer walls, and ground surfaces. The dimensions of the buildings including window areas and the nearby surroundings has to be specified in the calculations.

Shielding factors can be calculated for different photon energies and for a uniform surface activity distribution as well as for separate activity on roof, outer wall, and ground surface achieved from decontamination or different deposition velocities. For a given area with a known distribution of different houses a weighted shielding factor can be calculated ass well as a time-averaged one based on a given residence time distribution for work/school, home, outdoors, and transportation.

Calculated shielding factors are shown for typical Danish houses. To give an impression of the sensitivity of the shielding factors on the parameters used in the model, variations were made in some of the most important parameters: wall thickness, road and ground width, percentage of outer wall covered by windows, photon energy, and decontamination percentage for outer walls, ground, and roofs. The uncertainty of the calculations is discussed.

Availajle on request from Ris申 Library, Risø National Laboratory (R1s申 Bibliotek), Forsegsanlag Ris申), DK-4000 Roskilde, Dennark

Telephone: (02) 371212 , ext. 2262. Telex: 43116 